\title{
Data processing pipeline for Herschel HIFI ${ }^{\star}$
}

\author{
R. F. Shipman ${ }^{1,2}$, S. F. Beaulieu ${ }^{3}$, D. Teyssier ${ }^{4,5}$, P. Morris ${ }^{6}$, M. Rengel ${ }^{4,7}$, C. McCoey ${ }^{3}$, K. Edwards ${ }^{3}$, D. Kester ${ }^{1}$, \\ A. Lorenzani ${ }^{8}$, O. Coeur-Joly ${ }^{9}$, M. Melchior ${ }^{10}$, J. Xie ${ }^{5}$, E. Sanchez ${ }^{14}$, P. Zaal ${ }^{1}$, I. Avruch ${ }^{1}$, C. Borys ${ }^{6}$, J. Braine ${ }^{11}$, \\ C. Comito ${ }^{13}$, B. Delforge ${ }^{2}$, F. Herpin ${ }^{11}$, A. Hoac ${ }^{6,}$, W. Kwon ${ }^{1,16}$, S. D. Lord ${ }^{6}$, A. Marston ${ }^{15}$, M. Mueller ${ }^{1,2}$, \\ M. Olberg ${ }^{12}$, V. Ossenkopf ${ }^{13}$, E. Puga ${ }^{4}$, and M. Akyilmaz-Yabaci ${ }^{13}$
}

1 SRON Netherlands Institute for Space Research, 3584 CA Utrecht, The Netherlands e-mail: russ@sron.nl

2 Kapteyn Astronomical Institute, University of Groningen, 9747 AD Groningen, The Netherlands

3 University of Waterloo, Waterloo, ON N2L 3G1, Canada

${ }^{4}$ Herschel Science Center, ESAC, Villefranca, 28080 Madrid, Spain

5 Telespazio Vega UK Ltd for ESA, European Space Astronomy Centre (ESA/ESAC), Operations Department, Villanueva de la Cañada, 28692 Madrid, Spain

${ }^{6}$ IPAC, Mail Code 100-22, Caltech, 1200 E. California Blvd., Pasadena, CA 91125, USA

7 Max Planck Institute für Sonnensystemforschung, 37077 Göttingen, Germany

8 Osservatorio Astrofisico di Arcetri, 50125 Florence, Italy

9 IRAP, Institut de Recherche en Astrophysique et Planétologie CNRS/UPS, 31028 Toulouse, France

${ }_{10}$ FachHochschule Nordwest, 4600 Olten, Switzerland

11 Laboratoire d'Astrophysique de Bordeaux, Univ. Bordeaux, CNRS, B18N, Allée Geoffroy Saint-Hilaire, 33615 Pessac, France

12 Onsala Space Observatory, 43992 Onsala, Sweden

13 University of Cologne, 50674 Cologne, Germany

14 ESTEC, 2201 AZ Noordwijk, The Netherlands

15 ESA, The Space Telescope Science Institute, Baltimore, MD 21218, USA

16 Korea Astronomy and Space Science Institute (KASI), 776 Daedeokdae-ro, Yuseong-gu, 34055 Daejeon, Republic of Korea

Received 16 June 2017 / Accepted 12 July 2017

\begin{abstract}
Context. The HIFI instrument on the Herschel Space Observatory performed over 9100 astronomical observations, almost 900 of which were calibration observations in the course of the nearly four-year Herschel mission. The data from each observation had to be converted from raw telemetry into calibrated products and were included in the Herschel Science Archive.

Aims. The HIFI pipeline was designed to provide robust conversion from raw telemetry into calibrated data throughout all phases of the HIFI missions. Pre-launch laboratory testing was supported as were routine mission operations.

Methods. A modular software design allowed components to be easily added, removed, amended and/or extended as the understanding of the HIFI data developed during and after mission operations.

Results. The HIFI pipeline processed data from all HIFI observing modes within the Herschel automated processing environment as well as within an interactive environment. The same software can be used by the general astronomical community to reprocess any standard HIFI observation. The pipeline also recorded the consistency of processing results and provided automated quality reports. Many pipeline modules were in use since the HIFI pre-launch instrument level testing.

Conclusions. Processing in steps facilitated data analysis to discover and address instrument artefacts and uncertainties. The availability of the same pipeline components from pre-launch throughout the mission made for well-understood, tested, and stable processing. A smooth transition from one phase to the next significantly enhanced processing reliability and robustness.
\end{abstract}

Key words. instrumentation: spectrographs - methods: data analysis

\section{Introduction to the HIFI instrument}

The Heterodyne Instrument for the Far-Infrared (HIFI; de Graauw et al. 2010) was a heterodyne spectrometer that operated on board the Herschel Space Observatory (Pilbratt et al. 2010) between May 2009 and April 2013.

In heterodyne spectroscopy, the incident sky signal at frequency $v_{\text {sky }}$ is mixed with a locally generated signal (local oscillator, LO) at $v_{\mathrm{LO}}$ close to the sky frequency and passed onto

\footnotetext{
* Herschel was an ESA space observatory with science instruments provided by European-led Principal Investigator consortia and with important participation from NASA.

$\dagger$ Deceased September 2016.
}

a non-linear receiver. The non-linear receiver is sensitive to the frequency "beats" that the mixing produces. The beat frequencies $\left(\left\|v_{\text {sky }}-v_{\text {LO }}\right\|\right)$ cover a band of frequencies at a significantly lower frequency that can be readily analysed. A frequency within this band is called the intermediate frequency (IF) of the instrument. Although the IF is at a much lower frequency than the sky it retains the information of the sky signal. The IF band is then amplified and passed on to spectrometers. The HIFI receivers were dual sideband meaning that information at any $v_{\mathrm{IF}}$ was the combination of information at $v_{\mathrm{LO}}+v_{\mathrm{IF}}$ (upper sideband, USB) and $v_{\mathrm{LO}}-v_{\mathrm{IF}}$ (lower sideband, LSB).

HIFI was made up of 14 dual-sideband (DSB) receivers covering frequencies from $480 \mathrm{GHz}$ to $1906 \mathrm{GHz}$ in two 
polarisations (horizontal $(\mathrm{H})$ and vertical $(\mathrm{V})$ ). The LO produced continuous signal from 480 to $1272 \mathrm{GHz}$ in five continuous frequency bands (bands 1 to 5) and two high frequency bands between 1430 and $1906 \mathrm{GHz}$ (bands 6 and 7). The mixers which combined the sky signal and the LO signal were superconductorinsulator-superconductor (SIS) mixers for bands 1 to 5 and hot electron bolometer (HEB) mixers for bands 6 and 7. The IFs for receiver bands 1 to 5 covered frequencies from 4 to $8 \mathrm{GHz}$, while the IFs for bands 6 and 7 covered frequencies from 2.4 to $4.8 \mathrm{GHz}$.

HIFI was equipped with two spectrometer backends, the acousto-optical Wide Band Spectrometer (WBS), and the High Resolution Spectrometer (HRS) autocorrelator. For intensity calibration purposes, HIFI used internal hot and cold loads, and a chopping mechanism to choose between the loads and the sky-on target, and sky-3' off target. Extensive online HIFI documentation can be found in the Herschel Explanatory Legacy Library ${ }^{1}$ where a thorough overview of the HIFI instrument is documented in the HIFI Handbook (Teyssier et al. 2017, hereafter HB).

The conversion of the raw HIFI telemetry to scientifically usable products was performed by the HIFI pipeline. The HIFI pipeline and all associated data products were developed within the Herschel Common Software System (HCSS). The goal of the HCSS was to bring the three Herschel instruments together with the satellite into one common software framework. This framework had broad implications ranging from defining the underlining software language (Java) and databases to implementing common product definitions for images, spectra, and data cubes. It is within the HCSS that all instrument pipelines operated within the Standard Product Generation (SPG) component. Within this context, HIFI data products begin very HIFI specific and become more HIFI independent at the highest level of pipeline processing.

The pipeline was designed by the HIFI Instrument Control Centre (ICC) to process raw telemetry for the entire Herschel HIFI mission from the pre-launch laboratory phase to the post mission archive phase, a roughly $15 \mathrm{yr}$ period. To accommodate the many anticipated and unanticipated changes inherent in designing software over such a large time span, the pipeline was divided into processing levels comprised of (mostly) independent processing steps.

The HIFI pipeline produced data products for all HIFI observing modes as well as sensible processing for HIFI engineering modes (not discussed in this article). The pipeline also operated in both an interactive user environment for the calibration scientists and instrument engineers as well as within a completely hands-off environment of bulk processing (SPG).

During the mission, the HIFI instrument performed over 9100 observations where about $10 \%$ were calibration observations. The pipeline processed data from these observations are publicly available from the Herschel Science Archive ${ }^{2}$ (HSA).

The purpose of this article is to describe the HIFI standard processing pipeline for HIFI astronomical observations. This article focuses on the issues of the pipeline that mainly impact a general astronomer or archive user. A more detailed paper describing the implementation of the pipeline software will be presented in (Edwards et al., in prep., hereafter Paper II).

\footnotetext{
1 https://www . cosmos.esa.int/web/herschel/

legacy-documentation-hifi

2 http://www. cosmos.esa.int/web/herschel/

science-archive
}

This article is organised as follows. Section 2 reviews the observing modes which were available for the HIFI instrument during nominal operations. The relevant data processing concepts are described in Sect. 3. In Sect. 4, selected pipeline steps are described. Section 5 reviews the various quality assessment steps that are taken within the pipeline. Section 6 identifies a number of further processing steps beyond the pipeline which might be useful for full exploitation of the data. Section 7 reviews some of the main lessons learned in the process of developing a data processing pipeline. We end with a summary in Sect. 8 .

\section{Observing modes}

The HIFI pipeline is the implementation of the HIFI instrument calibration framework (flux calibration Ossenkopf 2003; and frequency calibration Herpin \& Teyssier 2003) and brings together instrument, satellite, and observation information that has bearing on the observational results. The main principle of observing with HIFI was to quickly switch between the astronomical source and some reference signal to correct for instabilities that were due to drift within the signal chain. To correct for standing waves, observations of a blank sky (OFF) were performed. The OFF measurements required nodding the telescope and were taken at a slower rate. For the faster referencing, HIFI made use of internal sources (hot and cold loads), modulation of the LO, or two chopped positions on (assumed) blank sky, each at a fixed distance of $3^{\prime}$ on either side of the source. The quick modulation measurements corrected for the spectral response of the system (bandpass), whereas the OFF measurement corrected for instrument response drift and standing waves.

The timing and frequency of the referencing are governed by the Allan instability time (Ossenkopf 2008) and the desired observation signal-to-noise ratio (Morris \& Ossenkopf, in prep.). Essentially, the timing within an observation was optimised to make efficient observations in the radiometric regime of the mixer.

The calibration reference schemes available to astronomers through the Herschel Observation Planning Tool (HSpot; Kidger 2013) are listed below.

- Position switch (PSW): this modulated the signal against an OFF position by slewing the telescope.

- Dual beam switch (DBS): this used the internal chopper to modulate the input signal to an OFF position $3^{\prime}$ from the source, then slewed the satellite $3^{\prime}$ to the other side of the source (nodding), and then it chopped back to the ON.

- Frequency switch (FSW): this modulated the LO to a frequency $\left(v_{0}+v_{\text {throw }}\right.$, where $v_{\text {throw }}$ can be positive or negative). The correction for instrument response drift was achieved by performing an FSW on an OFF position.

- Load chop: this used the internal chopper to modulate the signal against the internal cold load. The correction for instrument response drift was achieved by performing a load chop on an OFF position.

HIFI observed using astronomical observing templates (AOT) for different referencing schemes for the following three different modes: single spectra, spectral mapping, and spectral scans. These modes and their allowed referencing schemes are described below.

- Single Point mode: spectra taken at a single position on the sky in one LO setting were called "Point" spectra (sometimes referred to as single-point spectra). Point spectra could be observed using the referencing schemes described above. 
Table 1. Allowed CUSMODE/INSTMODE keywords for AOTs.

\begin{tabular}{|c|c|c|c|c|}
\hline AOT: CUSMODE name & Mode & Referencing scheme & OFF & Remarks \\
\hline HifiPointModeDBS & Single Point mode & Dual Beam Switch & $3^{\prime}$ & \\
\hline HifiPointModeFastDBS & Single Point mode & Dual Beam Switch & $3^{\prime}$ & Timing variant of DBS \\
\hline HifiPointModePositionSwitch & Single Point mode & Position Switch & yes & \\
\hline HifiPointModeFSwitch & Single Point mode & Frequency Switch & yes & \\
\hline HifiPointModeFSwitchNoRef & Single Point mode & Frequency Switch & no & \\
\hline HifiPointModeLoadChop & Single Point mode & Load Chop & yes & \\
\hline HifiPointModeLoadChopNoRef & Single Point mode & Load Chop & no & \\
\hline HifiMappingModeDBSRaster & Spectral Mapping mode & Dual Beam Switch & $3^{\prime}$ & \\
\hline HifiMappingModeFastDBSRaster & Spectral Mapping mode & Dual Beam Switch & $3^{\prime}$ & Timing variant of DBS \\
\hline HifiMappingModeOTF & Spectral Mapping mode & Position Switch & yes & \\
\hline HifiMappingModeFSwitchOTF & Spectral Mapping mode & Frequency Switch & yes & \\
\hline HifiMappingModeFSwitchOTFNoRef & Spectral Mapping mode & Frequency Switch & no & \\
\hline HifiMappingModeLoadChopOTF & Spectral Mapping mode & Load Chop & yes & \\
\hline HifiMappingModeLoadChopOTFNoRef & Spectral Mapping mode & Load Chop & no & \\
\hline HifiSScanModeDBS & Spectral Scan mode & Dual Beam Switch & $3^{\prime}$ & \\
\hline HifiSScanModeFastDBS & Spectral Scan mode & Dual Beam Switch & $3^{\prime}$ & Timing variant of DBS \\
\hline HifiSScanModeFSwitch & Spectral Scan mode & Frequency Switch & yes & \\
\hline HifiSScanModeFSwitchNoRef & Spectral Scan mode & Frequency Switch & no & \\
\hline
\end{tabular}

- Spectral Mapping mode: spectra taken at multiple positions at the same LO resulted in spectral maps. There were two main spectral mapping modes, raster and on-the-fly (OTF). The raster mode always used the DBS referencing scheme. In the OTF, the Herschel satellite scanned a pattern while HIFI integrated on the sky using one of the reference schemes, FWS, Load-Chop, or PSW.

- Spectral Scan mode: spectral surveys were obtained at a fixed position on the sky while scanning a wide LO range within a given mixer band and were performed in DBS, FSW, or Load-Chop referencing schemes. Position-switch referencing scheme was not available for this mode.

Table 1 lists the available AOTs (mode and referencing scheme) that were available for any given HIFI observation, and lists the mode names as they are found in archived HIFI data products. For historical reasons, the modes are identified as CUSMODE within the HIFI data products. A detailed description of each mode and referencing scheme can be found in Morris \& Ossenkopf (in prep.).

Observations are comprised of the source and OFF position, the frequency, the integration time (or desired noise), and the AOT. Together, these constituted an Astronomical Observing Request (AOR). Every scientific observation in the HSA is the result of an AOR. The HSA also contains calibration observations. Usually, these observations made use of standard observing templates, but they could have been taken with some different observing parameters. However, for automatic processing the fundamentals of these observations did not differ significantly from a standard AOT.

\section{Concepts of HIFI pipeline processing}

\subsection{One pipeline for all observations}

The HIFI pipeline is the component of the SPG (Ott 2010) that processed HIFI-specific data to populate the HSA. The pipeline was specifically designed for processing data on a polarisation basis from each of the spectrometers used in the observation. This can include low-level diagnostic housekeeping data even for a spectrometer that was not employed for science. The HIFI data were automatically processed through the pipeline and included in the HSA. All data were reprocessed during periodic bulk reprocessing after major software updates. The last bulk reprocessing for HIFI was completed with SPG version 14.1. About a dozen observations, however, had to be processed with SPG version 14.2 to fix an isolated issue.

Processing HIFI data makes best use of the fact that the same processing steps are needed regardless of observing mode and referencing scheme or spectrometer. For example, after the calibration of the different spectrometers, all processing steps are the same for either the HRS or the WBS data. The following sections contain pointers that describe where differences in the pipeline exist.

\subsection{Data format}

In the pre-launch phase, the main use of the pipeline was to process and test the spectrometer backend data. A HIFI data block consisted of the flux and frequency of all channels in a single readout of one of the backend spectrometers. An integration was built up of multiple readouts. Given the potential for instrument drifts, the readouts were not immediately co-added. The frequency values were not assumed to be fixed to a channel. At any one time, an instrument readout consisted of fluxes and frequencies at each channel of the spectrometer.

HIFI took observations as a function of time steps as prescribed by the Observing Mode command sequence that was generated to carry out the astronomer's AOR. At any moment, HIFI was executing the flow of the observation template, be it configuring the instrument, slewing the satellite, integrating on source or off source, or an internal load. At any one time, HIFI observed intensity as a function of frequency. When HIFI was performing the same sort of repeated integration, a twodimensional spectrum was being built over time. The container of such a spectrum was called a HifiSpectrumDataset. A given observation sequence could have hundreds or thousands of individual integrations. Instead of creating hundreds of thousands of individual spectra, the spectra were grouped in time sequences that formed 2D blocks of spectra. Each block belonging to a given activity as defined by the AOT. These blocks of 
spectra were placed into a HifiTimelineProduct. All HIFI processing was made on these $2 \mathrm{D}$ spectra. Listing spectra as $2 \mathrm{D}$ entities is a non-standard format that was not directly usable in most software packages for spectra. At the very end of the pipeline when all the observing mode specific time sequences of spectra were reduced, the resulting spectra were reformatted to a standard data format for spectra (frequency vs. 1D flux). Recently, GILDAS/CLASS (Bardeau et al. 2015; Bardeau \& Pety 2015) has been updated to read HIFI data directly in its native format.

All HIFI data come from the HSA in the form of a directorytree structure. The tree structure is a Java HCSS-specific set of product bindings and pointers to other products, objects, and metadata. When inspected in the Herschel interactive processing environment (HIPE; Ott 2010), main branches are referred to as context. A context is a special type of product that links other products in a coherent description and can be thought of as an inventory or catalogue of products. The HIFI-processed observation consists of many such contexts enclosed within one observation context. The observation context is therefore a product container that is comprised of various layers of science data, auxiliary data, calibration data, and other information about the observation.

MetaData form another important data component and describe each data product in more detail. MetaData contain parameters such as the source name, observation id, or the LO frequency, but also observation performance parameters. The MetaData perform the same function as FITS headers but for all products and sub-products. More detailed information on any HIFI data product is found in HIFI Products Explained (Beaulieu \& Teyssier 2017) $^{3}$.

\subsection{Calibration}

Calibrating HIFI data involved converting raw instrument outputs into scientifically usable quantities, that is, fluxes (antenna temperatures) and frequencies. It also entailed assessing the performance of the calibration steps, that is, whether were the resulting parameters where within the expected ranges. This last item required information from the AOR that defined the observation. To accommodate these concepts, the HIFI calibration was separated into three main sections:

- Downlink: these are pipeline input parameters that were measured independently of any one observation and were relatively constant over a span of time. Some example parameters are: load coupling coefficients and sideband ratios.

- Pipeline-out: these were pipeline-calculated parameters that were the results of pipeline steps, for example, the system noise temperature $\left(T_{\text {sys }}\right)$.

- Uplink: these were parameters from the AOR that specified how the observation was to be performed. The noise goals were specified in the uplink calibration.

When obtaining the Calibration product for an observation in the HSA, the retrieved data will reflect these three calibration areas and present the data either as FITS binary tables or spectra within each. A full description of the HIFI calibration tree is given in the Chap. 5.6 of the HB.

\footnotetext{
3 http://herschel.esac.esa.int/twiki/pub/Public/ HifiDocsEditableTable/HIFI_Products_Explained_v1.pdf in HIFI online documentation.
}

Two especially notable products in the pipeline-out calibration are the OFF spectra and the uncertainty table. These are described in more detail below.

\subsubsection{OFF spectra}

For each observation with a dedicated reference position observation, also called "OFF spectra", the resulting OFF spectra were calculated and attached to the pipeline-out calibration branch under the directory ReferenceSpectra. The OFF spectra are in the native HIFI FITS format (Sect. 3.2).

Since these spectra are generally useful to identify potential emission contamination in the blank sky (OFF position), the spectra are available as Highly Processed Data Products (HPDP $)^{4}$ from the HSA or via a dedicated link from the Herschel Science Centre. The HPDP data-set offers the additional advantage of providing OFF spectra as cubes for DBS raster maps and deconvolved spectra for spectral scans, which are more directly comparable to their $\mathrm{ON}$-source counterparts.

For DBS referencing, the OFF spectra are made from the difference of the two sky positions on either side of the source, whereas for other referencing schemes, the OFF spectra are the spectra of the OFF position. The position-switch referencing scheme uses the OFF position for the fast referencing, and the OFF observation itself does not have an independent reference. In this case, a referencing scheme for the OFF is made from the average of the cold-load data for that observation. Without the benefit of a double difference, the OFF spectra can display significant standing waves which can be addressed through interactive processing (see Sect. 6.1).

\subsubsection{Uncertainty tables}

The HIFI flux calibration uncertainty is broken down between the various components that enter the general calibration equation (Ossenkopf 2015). The HIFI pipeline implements the propagation of errors and provides uncertainty tables per polarisation and sideband for each observation. For each uncertainty component, the uncertainty is given as coefficients of a polynomial describing the possible dependency on the Intermediate Frequency (IF). We note that the uncertainties in the table apply directly to the Level 2 or Level 2.5 products calibrated on the $T_{\mathrm{A}}^{*}$ scale.

For spectral maps in particular, only one uncertainty budget table is provided per spectrometer, polarisation, and sideband because no distinction is made between the respective map points.

The uncertainty table of a given observation can be downloaded from the HSA by retrieving the Calibration product. The FITS table is found under the directory pipeline-out: Uncertainty: for instance for WBS. Similarly to the OFF spectra dataset, the uncertainty tables can also be retrieved as a standalone HPDP from the HSA, or at the link mentioned in the previous section.

\section{Processing levels}

The operation of HIFI was performed in predefined command blocks set by the AOT command logic built for the astronomer's AOR. Some command blocks performed standard instrument set-up steps where the processing is well known and the results can be checked for consistency. However, some of the command blocks were designed to integrate on an internal reference or on

\footnotetext{
4 http://www . cosmos.esa. int/web/herschel/ highly-processed-data-products
} 
Table 2. Bbtypes that can appear in HIFI data.

\begin{tabular}{rrrrrr}
\hline \hline bbtype & Type & Line & wbs & $\mathrm{h}$ & Details \\
\hline 6004 & comb & false & true & false & WBS-Zero-Comb \\
6005 & $\mathrm{hc}$ & false & true & true & HIFI-Calibrate-hot-cold \\
6601 & tune & false & false & true & HRS-tune-block-aot \\
6609 & tune & false & false & false & Magnet-tuning-block-aot \\
6613 & tune & false & true & false & WBS-attenuators-block \\
6014 & comb & false & true & true & WBS-Zero-FCal \\
6021 & science & false & true & true & HIFIContOffIntegration \\
6022 & science & true & true & true & HIFIContOnIntegration \\
6031 & science & true & true & true & HIFISlowChopOnIntegration \\
6032 & science & false & true & true & HIFISlowChopOffIntegration \\
6035 & science & true & true & true & HIFILoadChopOnIntegration \\
6036 & science & false & true & true & HIFILoadChopOffIntegration \\
6038 & science & true & true & true & HIFIFSwitchOnIntegration \\
6039 & science & false & true & true & HIFIFSwitchOffIntegration \\
6042 & science & true & true & true & HIFIFastChopOnIntegration \\
6043 & science & false & true & true & HIFIFastChopOffIntegration \\
\hline
\end{tabular}

the OFF position with the purpose of correcting instrument instabilities. The pipeline processed these command blocks as prescribed by the AOR assuming a "best case" scenario - best case being that the timing of references, OFFs, and all calibration activities were sufficient to correct the HIFI data. For a review of the performance of the AOTs, see Morris \& Ossenkopf (in prep.). In light of the potential for corrections to have been incomplete, the pipeline processed data up to reasonable stopping points, referred to as pipeline levels. These pipeline levels are described below. A series of pipeline flow diagrams are found in Figs. A.1 to A.5 for each level. A detailed description of all the pipeline steps can be found in the HIFI Pipeline Specification ${ }^{5}$ (HPS). Here, a brief description of each level is presented:

\subsection{Level 0}

Level 0 was the rawest form of HIFI-processed data available for inspection. It has been minimally manipulated to create the initial HifiTimelineProduct (HTP). These data contained all the readouts of the HIFI spectrometers plus satellite-pointing information associated with them, and have undergone several "sanity checks" to flag any incidences of "out of limit" housekeeping parameters.

Figure A.1 shows the flow of the Level 0 pipeline. The Level 0 pipeline created an HTP for each spectrometer used in the observation, which in turn contained a data set for each integration type in the observation. The chopper state was used at this stage to help identify and verify the integration type.

Level 0 contained many more data blocks than higher levels of processing. Integration types reported the activity that was executed, be it an internal load measurement or observing the reference position on the sky. The data types were recorded in building block ids, and these are listed in Table 2 and online in the section "Standard Observing Modes" of the HPS. The table indicates whether the data block resulted in science data or was a spectrometer calibration step and to which backend that step belonged. The column "line" specifies a science data block that is on-source ("true") or on the reference ("false"). The bbtype is also referred to as Bbid in the summary table of the HTP.

\footnotetext{
5 http://herschel.esac.esa.int/hcss-doc-15.0/load/ hifi_pipeline/html/hifi_pipeline.html
}

The bbtypes specified the activity of the HIFI instrument at any given time and were specific to both AOT and reference scheme (e.g. DBS bbtype have been shared between AOTs).

Level 0 contained a Quality product based on checks on the Level 0 data for telemetry issues common to both the WBS and the HRS (in the CommonTm product). For each spectrometer, the Quality product also contained checks on the data frame count and quality.

Additionally, information from the HIFI Uplink product, which contained information calculated by HSpot concerning how the observation should have been carried out (e.g., the requested noise in the final spectra or the spacing between scan legs in a map) was copied to the HTP metadata. Level 0 data have units of channel number ("wave" scale) and counts (intensity scale). These products are therefore of very little interest to most of the archive users.

\subsection{Level 0.5}

At this stage, the pipeline converted the raw spectrometer output into the basic spectral elements of frequency and flux as a function of time. Since the two spectrometers are fundamentally different, there were two separate pipelines: one for the WBS spectrometer (Fig. A.2), and one for the HRS spectrometer (Fig. A.3). Additionally, the $\mathrm{H}$ and $\mathrm{V}$ mixers are optically (semi-) independent and their output data in these respective chains were in their own processing thread as well.

The processing of the WBS data (Schieder et al. 2003) was mostly dedicated to processing the CCD channels of the four sub-bands. Each CCD array had pixels that were never illuminated. These pixels determined the "dark" for the array. As part of the standard operations, zeros and comb measures were obtained together. A zero measurement was constructed by interpolation in time between two measures where possible, and subtracted from the fluxes. Generated from the $10 \mathrm{MHz}$ master oscillator (Local Oscillator Source Unit, LSU), the comb signal is a series of stable frequencies at $100 \mathrm{MHz}$ steps. This comb signal is used to assign a frequency scale to the WBS CCD channels. A quality measure of this processing was retained in the Quality product for the WBS data. Other quality information relative to the CCDs was also retained. 
The Level 0.5 pipeline for the HRS was the implementation of the signal processing described in Belgacem et al. (2004). The HRS had 4080 autocorrelation channels that were able to provide up to four sub-bands per polarisation. The observer had a choice of configurations of the HRS depending on the desired spectral resolution: wide band, low, normal and high resolution modes. It was possible to place each sub-band independently anywhere within the 2.4 or $4 \mathrm{GHz}$ IF. The HRS processing split the telemetry into sub-bands depending on the HRS mode used. The autocorrelation functions per sub-band were fast-Fourier transformed resulting in flux at each channel and sub-band. Finally, frequency columns for each sub-band were created.

The result of the Level 0.5 pipeline was a time series of integrations per back-end spectrometer in instrumental counts as functions of frequency.

These products were removed from the observation as soon as the Level 1 products were successfully generated. They can, however, be regenerated interactively if needed.

The only product retained in the Level 0.5 was the quality information of the WBS-H and WBS-V comb, and zero quality checks which are stored in a Quality product.

\subsection{Level 1.0}

The purpose of the Level 1 pipeline was to flux-calibrate the HIFI data using the internal load measurements, and to apply the referencing scheme that was used for the observation. Additionally, the observed frequencies were placed into the LSR velocity frame for fixed targets, and in the reference frame of the moving targets for solar system objects (SSOs). At this level of processing, integrations taken at different times were then combined. The overall flow of the Level 1 pipeline is shown in Fig. A.4 and is discussed below.

The quality product at Level 1 contained the results of the phase checks made by the Level 1 pipeline for each spectrometer that was used in the observation.

The processing steps taken during the Level 1 pipeline were as follows:

- checkDataStucture, checkFreqGrid, checkPhases: the first part of Level 1 pipeline performed a series of sanity checks on the data structure, frequency grid, and phases. The reported chopper positions were checked to lie within the appropriate ranges. This step was used to verify that the data structure belongs to the specified AOT and raised a quality flag reporting discrepancies. checkFreqGrid created a product called FrequencyGroups in the Calibration product which contained the analysis of the LO groups. checkPhases performed an analysis of the patterns found in the sequence of science and load measurements to be consistent with the observing mode, specifically, whether the correct sequence of chopper positions, buffer values, $\mathrm{ON} / \mathrm{OFF}$ positions, or LO frequencies could be recovered in the observed data. Quality flags were raised when issues were identified.

- doFilterLoads: this step provided a means to reduce the standing waves that could be introduced from the internal loads. This step could reduce the 90-100 MHz standing waves in bands 1 and 2 on strong continuum sources, but had limited success (even degradation) in other bands. In the default pipeline, this task was disabled.

- mkFluxHotCold: this calculated the calibration to the antenna temperature scale $T_{\mathrm{A}}^{\prime}$ (that is, the antenna temperature before correction from the rearward beam efficiency losses)
(Kutner \& Ulich 1981) based on the hot/cold load measurements and created a load-calibration object. The calibration held two objects, one was the bandpass that calibrated to the $T_{\mathrm{A}}^{\prime}$ temperature scale:

bandpass $=\left(L_{\mathrm{h}}-L_{\mathrm{c}}\right) /\left[\left(\eta_{\mathrm{h}}+\eta_{\mathrm{c}}-1\right) \times\left(J_{\mathrm{h}}-J_{\mathrm{c}}\right)\right]$

the second object was the system noise temperature based on the load integrations and the measured load temperatures $\left(T_{\mathrm{h}}, T_{\mathrm{c}}\right)$,

$T_{\text {sys }}=\frac{\left[\left(\eta_{\mathrm{h}}+\mathrm{Y} \times \eta_{\mathrm{c}}-\mathrm{Y}\right) \times J_{\mathrm{h}}-\left(\eta_{\mathrm{h}}+\mathrm{Y} \times \eta_{\mathrm{c}}-1\right) \times J_{\mathrm{c}}\right]}{\mathrm{Y}-1}$

where $\eta_{\mathrm{h}}$ and $\eta_{\mathrm{c}}$ are the coupling efficiencies towards the hot and cold loads, $J_{\mathrm{h}}$ and $J_{\mathrm{c}}$ are the Rayleigh-Jeans equivalent radiation temperatures of the hot and cold loads at $T_{h}$ and $T_{c}$, respectively, and $L_{\mathrm{h}}$ and $L_{\mathrm{c}}$ are the load integrations in counts. The factor $\mathrm{Y}$ is the ratio of the load integrations, $\mathrm{Y}=L_{\mathrm{h}} / L_{\mathrm{c}}$. The variables, $L_{\mathrm{h}}, L_{\mathrm{c}}, J_{\mathrm{h}}, J_{\mathrm{c}}, \mathrm{Y}, T_{\text {sys }}$, and the bandpass are all functions of frequency and hence are arrays.

- doChannelWeights: this calculated and applied radiometric weighting to each channel. $w=t_{\text {integr }} / T_{\text {sys }}^{2}$, where $t_{\text {integr }}$ is the duration of each integration. For the purpose of the data weights, $T_{\text {sys }}$ needed to be interpolated in time between any two load integrations or the nearest in time if no bracketing data existed. The weights were further smoothed over 20 channels.

- doRefSubtract: the purpose of this step was to perform the first difference of the double-difference scheme, by subtracting the reference signal. This step ass highly dependent on the referencing scheme.

- For the DBS scheme, the chopped reference was subtracted by assuming an ABBA pattern (here, A and B means REF and ON respectively).

- For the fastDBS scheme, the chopped reference was subtracted by assuming an ABAB pattern.

- For the FSW scheme, the integrations that were shifted in frequency by the throw were subtracted. This scheme assumed an ABBA pattern.

- For the Load Chop scheme, the integrations on the cold load were subtracted from the target integrations. This scheme assumed an ABBA pattern.

- For PSW schemes, this step was not performed. No fast reference was taken in these schemes.

- mkOffSmooth, doOffSubtract: when the observation was taken at a dedicated OFF position, the OFF signal was subtracted from the source signal. DBS observations always had OFF source positions $3^{\prime}$ on either side of the source given by the telescope slew. In the case of Load Chop or FSW referencing schemes, the OFF data were smoothed by a width parameter depending on the HIFI band used. Table 3 lists the smoothing widths per LO of the OFFs in this case. Based on calibration tests, the smoothing widths were chosen to minimize the noise added back into the spectrum via the do0ffSubtract step. When the observation was an OTF map or a PointSpectrum mode with PSW referencing, the OFF samples were averaged and interpolated to the time of the ON observation, and subtracted. A calibration product called Baseline was created in the Calibration product and contained the baseline from the OFF positions.

- doFluxHotCold: this applied the flux calibration based on the load measurements by dividing the signal by the bandpass interpolated to the time of the signal integration. The 
Table 3. Off spectra smoothing widths for both $\mathrm{H}$ and $\mathrm{V}$ polarisations.

\begin{tabular}{llll}
\hline \hline Band & $\begin{array}{l}\text { Frequency } \\
\mathrm{GHz}\end{array}$ & $\begin{array}{l}\text { LoadChop } \\
\mathrm{MHz}\end{array}$ & $\begin{array}{l}\text { FSwitch } \\
\mathrm{MHz}\end{array}$ \\
\hline 1a-5b & $480-1280$ & 9.0 & 11.0 \\
$6 \mathrm{a}$ & $1420-1457$ & 30.0 & 11.0 \\
$6 \mathrm{a}$ & $1457-1459$ & 10.0 & 11.0 \\
$6 \mathrm{a}$ & $1459-1522$ & 30.0 & 11.0 \\
$6 \mathrm{a}$ & $1522-1570$ & 18.0 & 11.0 \\
$6 \mathrm{~b}$ & $1570-1655$ & 18.0 & 11.0 \\
$6 \mathrm{~b}$ & $1655-1710$ & 30.0 & 11.0 \\
$7 \mathrm{a}-7 \mathrm{~b}$ & $1710-1910$ & 18.0 & 11.0 \\
\hline
\end{tabular}

bandpasses were determined for each hot/cold measurement as described for the mkFluxHotCold step. These hot/cold measurements typically occurred at the beginning and the end of an AOR, but they could also occur during an AOR (depending on the stability of the mixer). The resulting bandpasses were linearly interpolated to the time of the source integration, or if the source integration was not bracketed by a hot/cold sequence, the hot/cold sequence nearest in time was used. We note that for the observing modes Load Chop and Frequency Switch, doFluxHotCold was applied before mkOffSubtract and doOffSubtract in order to reduce the noise.

- doVelocityCorrection: taking into account the satellite motion with respect to either the local standard of rest (LSR) or an SSO, this task corrected the frequency scale to either LSR or the appropriate SSO velocity. This task used the full relativistic corrections.

- doHebCorrection: when the observation was made in band 6 or 7, the task applied the correction for the electrical standing waves (Kester et al. 2014). The corrections were contained within the Downlink section of the Calibration product.

- mkFlagSummary: this produced a summary table of any flags (per channel or per integration) that were raised during the Level 1 processing. This information was fed into the Leve1 Quality product as FlagSummary.

At the end of the Level 1.0 pipeline, all science spectra were flux calibrated to the antenna temperature $T_{\mathrm{A}}^{\prime}$ and the intermediate frequency was adjusted to $V_{\mathrm{LSR}}$ reference (still in $\mathrm{MHz}$ ). At this stage, separate integrations were not co-added, and calibration spectra (e.g. hot/cold loads) were still present in the HTP.

\subsection{Level 2.0}

The Level 2 pipeline (Fig. A.5) contained spectra for each spectrometer that was used in the observation. Level 2 data were converted into $T_{\mathrm{A}}^{*}$ scale (in $\mathrm{K}$ ) and into sky frequency $(\mathrm{GHz})$. Owing to the DSB frequency degeneracy, products were generated on both the USB and LSB frequency scales. Spectra were averaged together for each spectrometer for each LO setting, and for each spatial position in the observation. This resulted in a single spectrum (for each spectrometer) for point-mode observation, in an individual LO setting for spectral scans, and individual spectra per position and LO setting for maps.

The processing steps taken during the Level 2 pipeline were as follows:

- mkRef: this step extracted emission in reference positions. For DBS modes, this step calculated the difference in emission between the two chop positions. The resulting spectra gave a rough idea of whether the proposer chopped onto a region with emission. This step populated the calibration pipeline-out with the OFF spectra.

- doCleanUp: Up to this point, very little had been removed from the time-line products. This initial step to the Level 2.0 pipeline removed spectra used for calibrations (e.g., WBS comb spectra).

- doAnntennaTemp: this converted data into antenna temperature $T_{\mathrm{A}}^{*}$ by dividing all spectra by the forward efficiency $\eta_{l}$. We note that $\eta_{l}$ is the combination of radiation and rearward spillover and scattering losses as defined by Kutner \& Ulich (1981). For all HIFI bands, this efficiency is 0.96.

- mkSidebandGain: this created a calibration object that contained the band and IF specific sideband gains that were to be applied to the given observation (Kester et al. 2017).

- doSidebandGain: this calculated upper and lower sideband spectra by dividing by the sideband gain determined in the mkSidebandGain step. This step created spectra that are listed as WBS_USB, WBS_LSB, HRS_USB, HRS_LSB, but note that the USB and LSB determinations are data taken from the intermediate frequency, and a LSB spectrum (for example) may have a USB spectral feature in it. Truly separating sidebands requires multiple LOs to have been used, and the deconvolution task to have been run (see HB Sect. 5.8.4). Furthermore, we note that sideband-gain correction is only applicable to line emission from a given sideband, so that the continua of spectra after this correction were typically a factor 2 higher than the true single-sideband continuum (see HB).

- convertFrequency: this step converted the frequency scales from IF (in MHz) into USB or LSB (in $\mathrm{GHz}$ ) scales. For HIFI bands 1-5:

$v_{\mathrm{USB}}=v_{\mathrm{LO}}+v_{\mathrm{IF}}, v_{\mathrm{LSB}}=v_{\mathrm{LO}}-v_{\mathrm{IF}}$.

For HIFI bands 6 and 7:

$v_{\mathrm{USB}}=v_{\mathrm{LO}}+v_{\mathrm{up}}-v_{\mathrm{IF}}, \nu_{\mathrm{LSB}}=v_{\mathrm{LO}}-v_{\mathrm{up}}+v_{\mathrm{IF}}$

where $v_{\text {up }}$ is an additional up-conversion frequency given by $10.4047 \mathrm{GHz}$ for horizontal polarisation and $10.4032 \mathrm{GHz}$ for vertical polarisation.

- mkFreqGrid: this step created a frequency grid with uniform spacing. For WBS this was set at $0.5 \mathrm{MHz}$. The frequency grid spacing for HRS is found in the metadatum channelspacing.

- doFreqGrid: this step resampled the original fluxes and weights onto the uniform frequency grid provided in the previous task. The task used the Euler scheme and conserved total power. As noted above, the data were separated into upper- and lower-sideband products. The result of this frequency re-sampling step may be that the upper and lower sideband spectra now differ in array length.

- doAvg: when the observing mode was not a spectral map, all spectra at a given LO and sideband were co-added. This step created products per spectrometer, polarisation and sideband. The averaging of spectra was performed without checking for standing waves or baseline issues. In some cases, it was not necessarily beneficial to co-add all the spectra. For this reason, an "expert-generated" User-Provided Data Product of non-averaged Level 2 spectra is provided through the $\mathrm{HSA}^{6}$.

\footnotetext{
6 http://www. cosmos. esa. int/web/herschel/ user-provided-data-products
} 
- doHpbw: when the observing mode was a spectral map, then the half-power beam width was added to the metadata of the observation and was used to define the convolution kernel during the re-gridding process in the Level 2.5. This guaranteed that the spectral map was made with the same assumptions that were available to the observer at the time of planning the observation.

- checkPlatforming: a quality check was performed on all WBS spectra to determine whether adjacent sub-bands were consistent with each other. Each WBS sub-band overlapped with its neighbour. When the difference in flux values in the overlap region was more than 1.3 times the noise, a platforming flag was raised and reported in the product as well as in the quality summary. We note that this check is all or nothing, that is, if only one spectrum showed the platforming, the flag was raised on the entire observation.

- mkRms: the pipeline also calculated what the noise was in the final spectra and compares this with the predicted HSpot results. The resulting value was placed in the Trend product of the observation. This step produced quality flags when the noise of polarisation data differed too much (greater than $20 \%$ ) or when the combine noise was significantly higher $(>30 \%)$ than the original HSpot prediction. Either of these flags appeared in the Quality Summary report for the observation only when the limit was exceeded. The noise was determined, ideally, on a "line-free" portion of a baselinecorrected spectrum. This automated step had to identify and mask any spectral feature that appeared as a spectral line and removed any baseline drifts. Furthermore, the spectra were smoothed to the desired smoothing width provided by the observer to plan the observation.

- doChannelFlags: using data from the Calibration tree, this task flagged data in which spurs were known or suspected to exist. This step was necessary for higher quality spectral survey results as these flags forced the deconvolution to ignore flagged data. The flags were also added to point spectra and spectral maps, but this was without consequences for any further data processing.

- mkUncertaintyTable: this step generated products containing a table of random and systematic uncertainty values for the observation based on uncertainties for sideband ratio, optical standing waves, hot/cold load coupling, hot/cold load temperature, planetary model, aperture, and main beam efficiency. The uncertainties are listed as percentages. A table is available for each spectrometer, polarisation, and sideband. The uncertainties are calculated based on Ossenkopf (2015).

The results of the Level 2 pipeline for non-mapping mode data were coadded spectra. These spectra are listed either on the USB or LSB frequency scale. The flux calibration is in $T_{\mathrm{A}}^{*}$.

\subsection{Level 2.5}

The Level 2.5 pipeline processed the Level 2 data products into final nearest-to-science-ready products as possible, in operations that depended on the observing mode. The products created at the end of the Level 2.5 were then used to create the stand-alone browse products (Sect. 5.1).

In the historical evolution of the pipeline, Level 2.5 processing was defined somewhat flexibly for each Herschel instrument to carry out value-added enhancements to the essential deliverables generated by the Level 2.0 pipeline. In other words, Level 2.0 products were absolutely required and scoped as such in terms of development resources starting during Instrument
Level Testing (ILT) in the pre-launch phase, whereas further processing needed to reach science-ready products that could be achieved in other known processing environments available to astronomers was given lower priority. However, with the maturing of the pipeline and accumulation of products from all of HIFI's available Observing Modes in the HSA as the mission progressed from Performance Verification into the Routine Phase, it became clear that Level 2.5 processing within the same HCSS environment was more and more necessary. The Level 2.5 processing step was created in order to validate both the lower level pipeline and the performance of the Observing Modes, and to provide end users with the products which turned out to be much more demanding than initially estimated.

Export of all Level 2.0 products along with necessary calibration tables for certain processing (such as in the construction of spectral cubes) had many practical limitations, and small program teams also had limited resources to develop or adapt other software packages, or manage the high volume of data being produced by each observation up to Level 2.0. This was generally true for users of any of the three Herschel instruments; for HIFI the resource demands and complexities were greatest for spectral scans and spectral maps. As a consequence, Level 2.5 steps were introduced relatively early in the mission, first for the spectral maps and spectral scans with robust algorithms for producing spectral cubes and sideband-deconvolved 1D spectra, and later for Point Mode spectra involving more simple operations requested by users. While the added value of this processing was clear to HIFI scientists for iterative improvements to the underlying pipeline and AOT logic, the effort was further encouraged by the Herschel User Group and the Data Processing User Group.

Since the tasks that created Level 2.5 products were considerably more algorithmically complex than the individual tasks in the lower pipeline, especially for Mapping Mode and Spectral Scan observations, it is worthwhile to elaborate on their implementations.

\subsubsection{Point Mode}

Level 2.5 processing of Point Mode spectra was essentially a conversion of formats for ease of handling by end users. The spectra were sub-band stitched (doStitch), folded (in the case of Frequency Switch) into a single 1D spectrum, and converted into the so-called SimpleSpectrum format, again on a spectrometer, sideband, and polarisation basis. WBS spectra had IF sub-bands with overlaps of roughly $150 \mathrm{MHz}$ and were always stitched. Each sub-band was cut at a cross-over point that was generally the mid-point of the overlap; other options were available in HIPE. This resulted in a single spectrum, which simplified its handling by end users, but it lost information on potential sub-band-specific effects such as platforming (see $H B$ ). HRS data were stitched only when the sub-bands substantially overlapped in frequency, which was not always the case since this depended on the requested resolution mode and placement of the HRS sub-bands by the user during observation planning.

The stitched spectral data were populated into a so-called Spectrum1d, with columns for wave (frequency), flux (antenna temperature), flagging, weights, and segment number (set to unity after stitching). The data were contained in the HIFI data tree as SimpleSpectrum products, which are basically metadata wrappers around the Spectrum1d with links or "bindings", and pointers to other parts of the data tree. The SimpleSpectrum product definition was intended to allow flexibility in HIPE software applications for spectra produced by all three instruments. 


\subsubsection{Mapping mode}

Spectral mapping observations carried out at various single-dish ground-based receivers are typically provided to users as spectral cubes built up from planes of signal projected into celestial coordinates at each frequency channel, thus there do exist different software environments that might conceivably carry out the construction of the cubes with input Level 2.0 HTPs. The conversion of formats from the very instrument- and HCSS-specific HTPs into required file types such as standard FITS or CLASSFITS (for example) is non-trivial, however. Furthermore, in order to facilitate all of pipeline and AOT logic optimisations iteratively and self-consistently within the same processing environment, and fill a requirement by end-users for this key science product, the construction of spectral cubes was the first extension of the Level 2.0 pipeline into Level 2.5, and initially integrated into the SPG already during the Performance Verification phase (although still defined as Level 2.0 at that time).

The Level 2.5 processing for mapping observations began with sub-band-stitching of the Level 2.0 HTPs, that is, the individual spectra corresponding to different map points on the source were treated as Point Mode spectra and stitched as described above. Similar to Point Mode observations, stitching was always made to WBS spectra, and made to HRS spectra (if present) only when the sub-bands overlapped. The Level 2.5 HTPs are provided as products in the HIFI data tree, and constitute the inputs for the pipeline doGridding task that creates spectral cubes on a spectrometer (WBS/HRS), sideband (USB/LSB), and polarisation (H/V) basis.

The basic purpose of the doGridding task was to place all mapped on-source data from a Level 2.5 HTP into a regular spatial grid as a function of frequency. The algorithm is very similar to that used for mapping observations at the $30 \mathrm{~m}$ IRAM telescope, in which the globalized sinusoid (GLS) projection method (Calabretta \& Greisen 2002) provides the convolution of individual spectral readouts into effective "pixels" (sometimes referred to as "spaxels" for spatially synthesised pixels in non-imaging devices). In the case of HIFI, the pixel sizes were usually determined by stored parameters that reflect how the readout pattern was commanded on the sky, specifically, the number of scan lines, number of readout points per scan line, and the requested sampling density (Nyquist, half-beam, 10", 20", or $40^{\prime \prime}$ readout spacing). This information was not available for all observations, mainly from the early part of the mission, and in these cases, the task attempted to determine the nominal sampling from the attitude assignments to the spectra in the Level 2.5 HTPs. In both cases, after the initial-guess dimensions were determined, all spectra which fall spatially within the convolution kernel were convolved to fill each rectangular pixel.

The default convolution was an azimuthally symmetric Gaussian kernel based on the half-power beam width (HPBW). Values for the HPBW are provided in a look-up table in the observation's Calibration product at several frequencies in each mixer band (summarized in Table 4) based on flight beam calibrations (Mueller et al. 2014), and interpolated to the LO frequency of the observation. The 2D Gaussian function in $x$ and $y$ positions (i.e., indexed map position) is expressed as

$G(x, y) \propto \exp ^{-\left(\left(x^{2}+y^{2}\right) / 2 \sigma^{2}\right)}$.

The pipeline used a kernel sigma $\sigma_{k}=0.3 \times \sigma_{\mathrm{B}}$ where $\sigma_{\mathrm{B}}=$ $H P B W /(2 \sqrt{(2 \ln 2)})$. The factor 0.3 results in a slightly larger (4\%) final beam in the regridded data cube. While the spacings $\Delta x$ and $\Delta y$ between map points were set to be equal in the AOT logic, determined only by the requested sampling and the beam
Table 4. Adopted values for $\eta_{\mathrm{mb}}, \eta_{\mathrm{A}}, H P B W$, and point-source sensitivity $S / T_{\mathrm{A}}^{*}$ (i.e., Kelvin to Jansky conversion factor) $=\left(2 k_{B} / A_{g}\right)\left(\eta_{l} / \eta_{\mathrm{A}}\right)$ for one spot frequency per mixer.

\begin{tabular}{crrrrr}
\hline \hline Mixer & $f$ & $\eta_{\mathrm{mb}}$ & $\eta_{\mathrm{A}}$ & $\begin{array}{r}H P B W \\
\prime\end{array}$ & $\begin{array}{r}\text { K-to-Jy } \\
\mathrm{Jy} / \mathrm{K}\end{array}$ \\
\hline $1 \mathrm{HHz}$ & 480 & 0.62 & 0.65 & 43.1 & 482 \\
$1 \mathrm{~V}$ & 480 & 0.62 & 0.63 & 43.5 & 497 \\
$2 \mathrm{H}$ & 640 & 0.64 & 0.64 & 32.9 & 489 \\
$2 \mathrm{~V}$ & 640 & 0.66 & 0.66 & 32.8 & 474 \\
$3 \mathrm{H}$ & 800 & 0.62 & 0.63 & 26.3 & 497 \\
$3 \mathrm{~V}$ & 800 & 0.63 & 0.66 & 25.8 & 474 \\
$4 \mathrm{H}$ & 960 & 0.63 & 0.64 & 21.9 & 489 \\
$4 \mathrm{~V}$ & 960 & 0.64 & 0.65 & 21.7 & 482 \\
$5 \mathrm{H}$ & 1120 & 0.59 & 0.54 & 19.6 & 580 \\
$5 \mathrm{~V}$ & 1120 & 0.59 & 0.55 & 19.4 & 569 \\
$6 \mathrm{H}$ & 1410 & 0.58 & 0.59 & 14.9 & 531 \\
$6 \mathrm{~V}$ & 1410 & 0.58 & 0.60 & 14.7 & 522 \\
$7 \mathrm{H}$ & 1910 & 0.57 & 0.56 & 11.1 & 559 \\
$7 \mathrm{~V}$ & 1910 & 0.60 & 0.59 & 11.1 & 531 \\
\hline
\end{tabular}

Notes. This table is to be compared to Table 5 in Roelfsema et al. (2012), which uses the same spot frequencies, but outdated efficiencies and beam widths.

size at the associated LO frequency, deviations from the planned path of the telescope occurred because neither the actual nor reconstructed telescope points were errorless.

The contribution of data points near a spatial pixel was determined by the Gaussian-weighted average of all data within an influence length. Data points that were within an influence length of $3 \times \sigma_{k}$ in both $x$ and $y$ from the centre of the pixel were convolved. The weight factor $G_{n}(x, y)$ for the $x, y$ pixel from data point $n$ at the grid position $x_{n}, y_{n}$ is:

$G_{n}(x, y)=\exp ^{-1 / 2\left(x-x_{n}\right)^{2} / \sigma_{k}^{2}} \times \exp ^{-1 / 2\left(y-y_{n}\right)^{2} / \sigma_{k}^{2}}$

where $x_{n}$ and $y_{n}$ are the positions of the observed data point $n$.

The convolution combines data within an influence length. When $N$ data points fall within this length for a pixel at $(x, y)$, the flux is given as convolution of the data fluxes, $F_{n}$ :

$F(x, y)=\Sigma_{n=1}^{N}\left(G_{n}(x, y) \times F_{n}\right) / \Sigma_{n=1}^{N} G_{n}(x, y)$.

Figure 1 shows an example of six beam positions (red circles) on a grid, where two positions are within the influence area (blue square).

Each spectrum from the input HTP was not necessarily unique in terms of its celestial coordinates; more than one spectrum per map point was produced in cases where the observation was carried out over more than one mapping cycle, driven by baseline noise goals during observation planning. No two spectra taken at the same map point had identical coordinates because of uncertainties in the actual and reconstructed telescope pointing, but the differences for spectra taken at the same point were usually small compared to the HPBW and tended to be noticeable mainly in maps taken with the OTF mode. An example is shown in Fig. 2. The positional differences between spectra at the same map point, as well as slew deviations from the ideal path of the telescope during mapping such as the OTF "zig-zag" or "hula dance" effect (Morris 2011, 2015), were taken into account in the weighting filter profile 


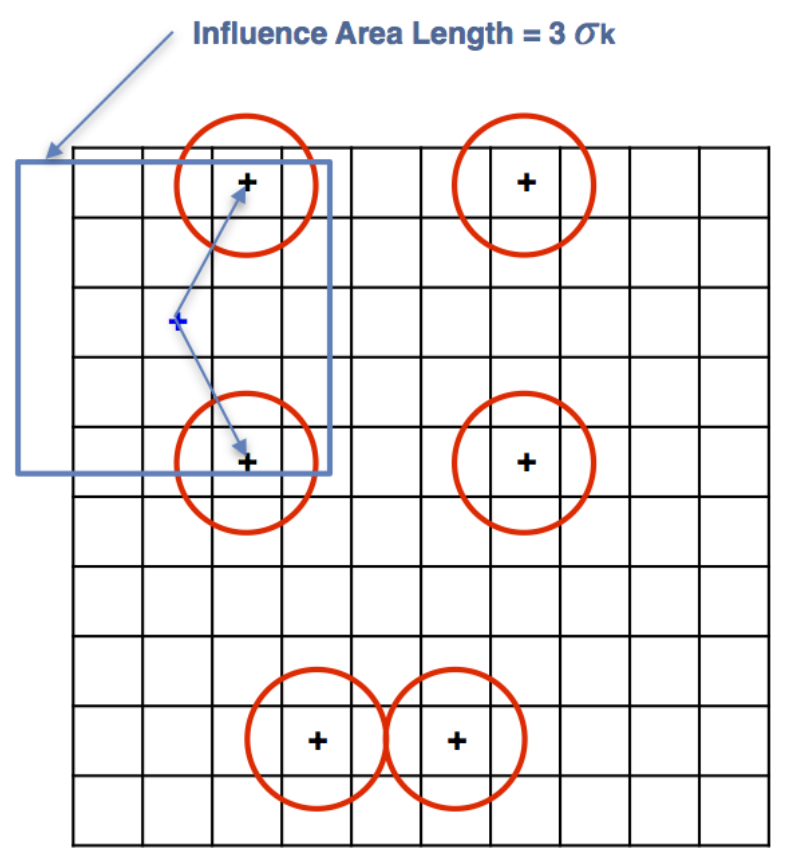

Fig. 1. Schematic representation of the convolution of spectral map points onto a regular grid by the doGridding task. The pluses represent map readout points with the HPBW represented by red circles, and the blue squares represent an influence area.

during convolution. These deviations have had some important consequences:

- Throughout most of the mission, the pointing reconstruction did not accurately reflect the anomalous telescope performance of the line-scan-with-off pointing mode used for OTF mapping, which led to the apparent "zig-zag" or alternating shift of pixels on each scan line in the gridded spectral cubes. The effect was strongest in HIFI's high-frequency bands (smallest beams). The effects were greatly reduced after the drift from the four gyroscopes on the telescope was characterised more accurately, leading to a more accurate pointing reconstruction and thus signal weighting during cube construction (in most cases).

- The gridded pixels were not always of equal size on each axis because of the general pointing performance uncertainties. In many observations the effects were essentially averaged out, and pixels were very close to square, the differences being much smaller than a fraction of the beam size. Some small maps that had only a few map points, or maps that were taken in the early part of the mission, were particularly susceptible to variations in angular length in one or the other dimension. The example observation shown in the bottom panel of Fig. 2 has slightly rectangular (non-square) pixels for this reason, where the slight deviations seen on close inspection in the upper panel are not completely averaged out in both dimensions on the sky.

We note that the HIFI beams were only used for weighting in the convolution kernel, and for this purpose the approximation of the beams as 1D symmetric Gaussian profiles was adequate. The doGridding task does not have any capability to deconvolve the beam shapes from the signal in the constructed cubes. This operation was considered a worthwhile augmentation of the task in interactive applications, but ultimately this functionality
1342210097 apid[1030] sky positions
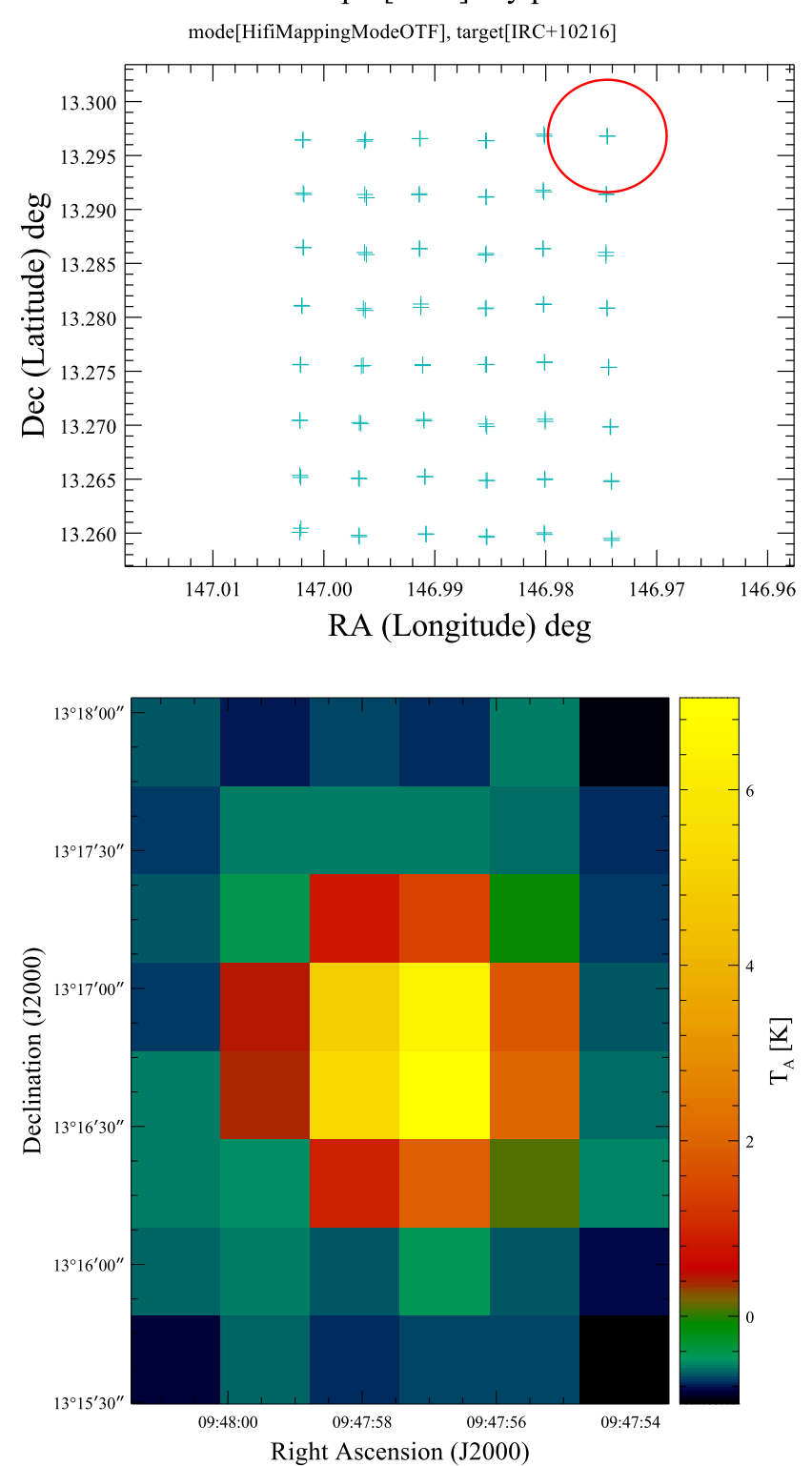

Fig. 2. Top: sky positions for map points of an OTF map (obsid $1342210097)$ of IRC +10216 with the LO tuned to USB coverage of $\mathrm{CO} J=5-4$ in band $1 \mathrm{~b}$. The pluses represent the attitudes read from the PointingProduct and assigned to each spectrum data-set in the Level 1 pipeline. Two map cycles using half-beam sampling were carried out in this observation. The size of the beam $\left(H P B W=37^{\prime \prime} .5\right)$ is shown in red. Bottom: map gridded into square pixels for the same observation. Each pixel is $19^{\prime \prime} .5 \times 19^{\prime \prime} 2$, i.e., not perfectly square because of overall pointing errors that do not average out in the entire map.

was not developed because of system software stability risks and resource limitations.

At this stage, the processed spectral cubes were added to the HIFI data tree under a Level 2.5 "cubesContext". The cubes were readily operated on by tasks in HIPE and exported to FITS. A second set of cubes was produced in the SPG with the doGridding task for maps that were taken at a non-zero rotation angle (i.e., not square with RA and Dec). In this case, the GLS algorithm was applied at the specified rotation angle to project the map into a filled square map with generally non-square pixels correctly projected in celestial coordinates such that signal was conserved (theoretically) compared to the pixels constructed at 
native orientation. These cubes, similarly generated on a spectrometer, sideband, and polarisation basis, were populated into a "cubesContextRotated" Java construct.

It should be noted that the doGridding task in the SPG assumed equal quality of all spectra within the input HTP. However, when any frequency channel(s) of the input spectra was flagged for quality problems, such as possible spurs, saturation, or unruly conditions of the WBS CCD pixels spreading across many channels, the task gave zero weighting to the affected channels during signal convolution. This could result in an NaN assignment to the signal at the associated frequencies in some or all pixels, unless more than one map cycle was taken and the problem was transient and not systematic over the entire observation.

The doGridding Application Program Interface (API) included extensive top-level parametrisation to allow flexible user interaction with the task in HIPE. This was somewhat different in the lower level pipeline tasks - although most of the tasks were parametrised for customisable processing in HIPE, intervention in the Level 0 to 2.0 pipeline by users has been rarely needed (and in these rare cases usually involved rerunning the pipeline in a default configuration that used updated calibrations or bug fixes between major releases of the HCSS or bulk reprocessings in the HSA). The most common customisations of spectral cubes involved resampling to a different pixel size, to a finer grid when signal-to-noise ratios around a target spectral line were high, for example, or using different filter parameters to mimic the beam properties in mapping data obtained with other instruments, or matching the WCS of another observation to a HIFI mapping observation with spatial overlap. The task also had to be re-run in order to reach optimum baseline noise performances using input HTPs constructed with the mergeHtps task to combine data sets from the two polarisations, or from different observations. These customisable features were exploited to produce the mapping HPDPs available from the HSA (see footnote 4).

\subsubsection{Spectral Scan mode}

The concept of the Spectral Scan observing mode was that sufficient redundancy was built in sky frequency coverage in order to resolve the double-sideband degeneracy intrinsic to the HIFI detectors. The higher the redundancy, the more accurate the single-sideband spectrum that was reconstructed from the double sideband Level 2 spectra. The sideband deconvolution was performed for each polarisation, following the conjugate gradient method (Comito \& Schilke 2002). In this process, all artefacts present in the Level 2 spectra inputs were propagated into the final deconvolved spectrum. This was particularly critical for narrow spectral spurious features, which polluted the end results. This required careful masking of those spurious artefacts as one of the pipeline steps before the sideband deconvolution. Such masks were manually assigned for each individual spectrum involved in one of the nearly 500 Spectral Scans taken with HIFI. The masks were stored as calibration files and attached as flags to the spectra, and they were subsequently used to identify spectral channels to be ignored in the construction of the deconvolved spectrum.

Another fundamental parameter of the sideband deconvolution was the sideband gain to be applied to the respective sidebands. In the HIFI pipeline, this gain was tabulated as a calibration parameter and was applied to the Level 2 spectra as a function of their LO frequencies. This means that the gain had to be undone by the deconvolution algorithm, and then applied to the different spectral building blocks used to construct the single-sideband solution. In an earlier version of the deconvolution task coded for HIFI, an option was implemented that would attempt to actually fit the sideband gain based on the redundant information held by the Level 2 spectra. This approach, however, proved to provide inaccurate gains and was removed in the end. Instead, the chosen approach was to use sideband gains determined independently (Kester et al. 2017) as preset value to then apply in the task.

It should be noted that the deconvolution code implemented for HIFI was initially inspired by the code implemented in the GILDAS/CLASS software. Features such as channel masking and ad hoc sideband gain usage were, however, added as extra functionalities to the initial CLASS implementation. It is now possible to benefit from the masking effort in HIFI spectra imported into CLASS, for instance, see Bardeau et al. (2015) for further details.

The above processing guaranteed the best possible deconvolved solution from the calibrated Level 2 spectra, which was provided as Level 2.5 spectra. However, this does not cover any residual instrument artefact that could manifest as baseline distortion or ripples. For this purpose, a fraction of the HIFI Spectral Scans was further processed using dedicated baseline cleaning. These spectra were provided as Highly Processed Data Products (see footnote 4).

\subsection{Testing and validation of the pipeline}

Major components of the pipeline (Level 0 and Level 0.5) have been in active use since the pre-launch instrument models of HIFI; hence, all the products have a metadatum named modelName with the value FLIGHT. Before launch, Level 0 and Level 0.5 had been tested and validated, and extensive unit tests for all Level 1 and Level 2 processing tasks had been available and were based on regression data constructed in accordance with the expected behaviour of the instrument. However, only with the full integration on the satellite, could the later stages (Level 1 and Level 2) of the pipeline be truly tested. These stages were validated as part of the Performance and Verification phase of the HIFI mission and led to the release of standard modes of observing with HIFI (Morris 2011). The performance of the HIFI instrument is described in Teyssier (in prep.). Further testing and validation occurred periodically as the HCSS software was upgraded. The pipeline results also underwent significant testing during the mission for every major software upgrade of the Herschel ground segment. This verification comprised testing of new functionalities as well as regression testing. All modes were tested. Only when all problems had been fully addressed would the new version of the software be accepted to be released.

\section{Assessing data quality}

There are two main questions regarding data quality: (1) whether each processing step performed within nominal bounds and; (2) whether the AOR fully mitigated the instrument instabilities. The first question was monitored during the processing by retaining quality measurements during the processing of each step. Section 5.3 describes quality flags when one of the processing steps was out of limits.

The second quality assessment comes partly from the pipeline by measuring the resulting noise and comparing with the expected noise, but mainly from the astronomer who should visually inspect the pipeline products. The pipeline allows for 


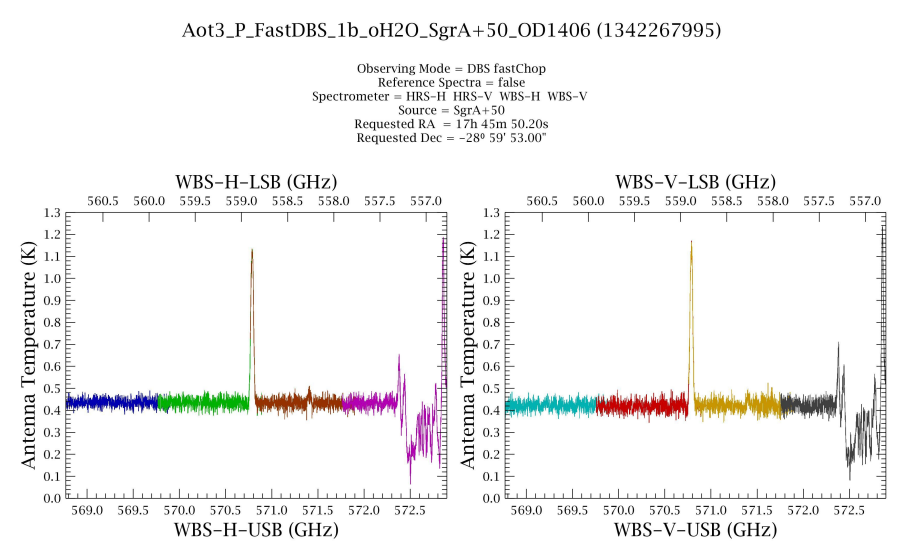

Fig. 3. Example of a browse image postcard provided with a HIFI DBS observation. The image is made from the observation WBS data, in each polarisation. The different colours are the WBS sub-bands. HRS data are not shown in the postcard.

inspection by providing products at all processing levels, and browse products at the final processing stage (Sect. 5.1). In Sect. 5.2, the priority of inspecting beyond the browse products is suggested.

\subsection{Browse products}

The final results of the HIFI pipeline processing were provided in two related products. The first was the stand-alone browse product itself. This product is the spectra or spectral cubes for each HIFI spectrometer of the observation as they are found at the end of the Level 2.5 processing.

The second product is the browse image or postcard. This image offered a quick look at the data. It corresponded to the postcard displayed in the HSA User Interface (HUI) and showed the outcome of the Level 2.5 product generation. We note that these products were not necessarily science-ready. Although these postcard images gave a first impression of the quality of the data, often the browse images showed problems that needed to be addressed with further processing (e.g. baseline ripples). A high-resolution image of the browse product is available by downloading the jpg product (right-click on the postcard).

For Single Point mode, the postcard shows some of the main observation parameters together with two plots of unstitched Level 2 WBS spectra with the H-polarisation to the left and the $\mathrm{V}$-polarisation to the right. Each browse image lists the uppersideband and the lower-sideband frequency scales. HRS spectra are not shown. Unless the spectral line is very strong, the images at first glance only show noise. The browse image does give a general impression whether the baseline in the data is flat, or not. Figure 3 shows an example of a browse product image. Here, we note that the apparent absorption feature $(572.3-572.8 \mathrm{GHz}-$ USB scale) is real and due to the 557 water line in the lower sideband. The Level 2.5 (see Sect. 4.5) data are downloadable with the browse image for more careful inspection.

For Spectral Mapping mode, the browse image shows sets of map-averaged Level 2 spectra for each sub-band along with the integrated map for that sub-band. For each sub-band, there are two sets of images, one for each polarisation. The spectra are formed by averaging all spectra per map position while the integrated map is the full integration over the entire sub-band. The spectra and integrated maps were created without correcting for any baseline problems (Sect. 6). When the baseline suffered from drifts and/or standing waves, the continuum dominates the map. The stand-alone browse data associated with the browse image are the spectral cubes with merged sub-bands per polarisation and spectrometer (see Sect. 4.5). Figure 4 shows an example of the browse image for cubes. At the top of the images, the observation is described briefly.

For Spectral Scan mode, the browse image shows the singlesideband solution after deconvolving the Level 2 WBS spectra. No baseline correction was made before deconvolution. The $\mathrm{H}$-polarisation is shown to the left and the V-polarisation to the right. The gap between the sidebands is shown as a line at $0 \mathrm{~K}$. Figure 5 shows an example of a browse image of a spectral scan. We note that the images are at significantly lower resolution than the information present in the data, and the images only provide a rough indication of what the data can reveal.

\subsection{Priority of inspecting pipeline products}

Although the stand-alone browse products give a good indication of the overall data quality, for science purposes the co-added spectra that make up the browse products might wash out isolated artefacts or add data deemed unusable for science. It is recommended that individual spectra that make up the browse product be inspected before scientific analysis.

For Single Point mode observations, Level 2 spectra correspond to the co-addition of all individual spectra, and reflect problems that occurred throughout most of the integrations. Level 1 should be inspected only to identify problems with any specific integration. If any are found, it is recommended to work from Level 2 data before to the co-addition. This can be achieved by a particular reprocessing of the Level 1 data, but is also readily available via the HSA as HIFI Non-averaged Level 2 Spectra ${ }^{7}$ for all Single Point mode observations.

For the Spectral Mapping and Spectral Scan modes, inspecting each Level 2 spectrum should give a good idea as to whether standing waves or other instrument artefacts are still present in the final products. When this is the case, the data can be baseline corrected and will then need to be combined again into either spectral cubes (for mapping) or deconvolved spectra (for spectral scans).

Alternatively, a fraction of Spectral Mapping and Spectral Scan modes have been manually cleaned from baseline artefacts by instrument experts, and can be fetched as Highly Processed Data Products (footnote 4) from the Herschel Science Archive.

\subsection{Quality reports}

In automatic processing, most processing steps will have an expected outcome. When the outcome is noticeably different than expectations, the pipeline will raise a flag. Some of the differences are readily identified (e.g., a failure to reach a solution to a WBS comb measurement). Others, like comparing noise in an observation with respect to predicted noise, are more involved and require significant processing of the observational results. The resulting quality flags should then be taken as an indication of potential problem and not necessarily a true problem. In any event, it is worth the effort to examine the data. An overview quality report is provided by the HSA and in the Quality Control Summary Report in HIPE for each observation and provides an adequate starting guide for potential data problems.

\footnotetext{
7 http://herschel.esac.esa.int/twiki/pub/Public/ HifiDocsEditableTable/HIFI_nonAveragedLevel2_UPDP_
} ReleaseNote.pdf 


\title{
Calibration_PM_12-Aot2_M_OTF_4a_H2O_524-431_OrionKL (1342190889)
}

\author{
Observing Mode $=$ OTF - Position Switch \\ Reference Spectra $=$ false \\ Spectrometer $=$ HRS-H HRS-V WBS-H WBS-V \\ Source = Orion small map \\ Requested RA $=5 \mathrm{~h} 35 \mathrm{~m} 14.50 \mathrm{~s}$ \\ Requested Dec $=-5^{\circ} 22^{\prime} 0.00^{\prime \prime}$
}
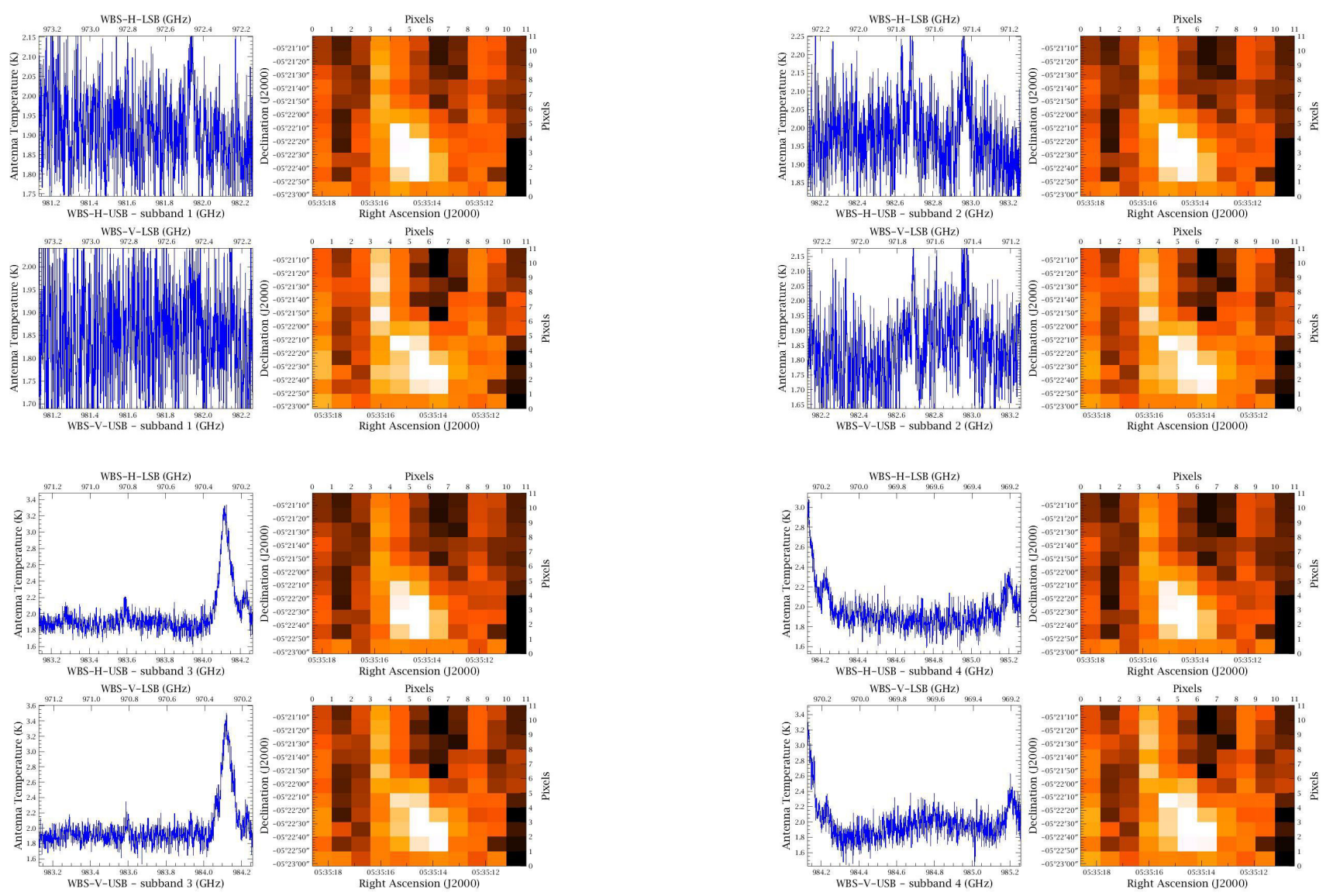

Fig. 4. Example browse images for a spectral cube. The image shows spectral cube data separately for each WBS sub-band and polarisation in the observation. The colour images are made by integrating over the frequencies in each sub-band, whereas the spectra are taken as an average over all positions. Top left panel: WBS sub-band $1 \mathrm{H}$ polarisation with $\mathrm{V}$ polarisation data just below. Top right panel: sub-band 2, bottom left panel: sub-band 3, and bottom right panel: sub-band 4.

Significant effort has been made to provide clear flag names and/or descriptions. At each level of processing quality items are reported. Chap. 10, Sect. 4 of the HIFI Data Reduction Guide (HDRG see footnote 1) provides a complete listing of all quality flags and actions suggested to be taken. Each flag includes a description of the detected problems, an evaluation of the effect on science data, and a recommendation for how to proceed in case that the flag is raised. The quality flags are separated into three levels of severity but these levels are not reflected in the Quality Summary Report provided to the user via the HSA. We note that the Quality Products provided with each level of data processing are the results of checks and are always populated with information even if nothing is found to be wrong whereas the Quality Report lists items that were flagged as problems.

\section{Additional processing}

For the majority of HIFI observations, Level 2 and Level 2.5 products can immediately be used for scientific analysis. However, as mentioned earlier, they were produced under the assumption that the referencing scheme sufficiently addressed all instabilities without additional correction for baseline drift or standing waves. Furthermore, the temperature scale or reference frame of the data may not be the most appropriate for a given scientific objective, such as combining HIFI data with other telescope data-sets.

Within the interactive HIPE software package, the HIFI pipeline provides a mechanism to perform some additional steps. The "Configurable Pipeline" feature of the hifiPipeline provides a way to rerun all or part of the pipeline in order to omit tasks or to change task defaults. Alternatively, it is possible to examine the results of each step of processing in order to improve data quality by accessing the ICC pipeline scripts used in the pipeline. It is also possible to use a "non-ICC" pipeline algorithm that is based in part on the ICC algorithm. Section 5.4 of the HDRG provides the necessary documentation on how to use the interactive hifiPipeline.

The observation that is obtained from the HSA contained, along with the Level $0-2.5$ data products, everything that is required to reprocess observations: calibration products, satellite 

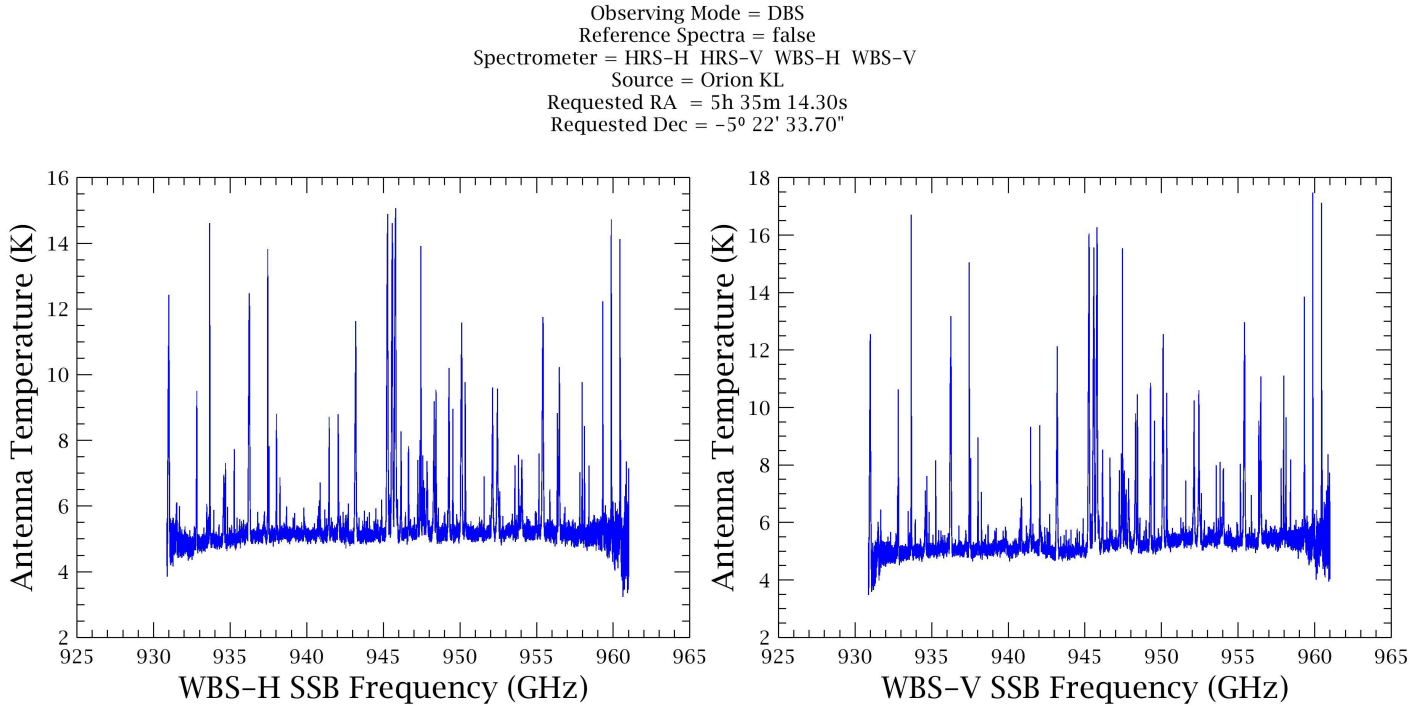

Fig. 5. Example browse images for a spectral scan. The data are the single-sideband spectra that resulted from the deconvolution. The two spectra show the $\mathrm{H}$ and $\mathrm{V}$ polarisations, respectively. The frequency range reflects the frequencies that are covered in the spectral scan.

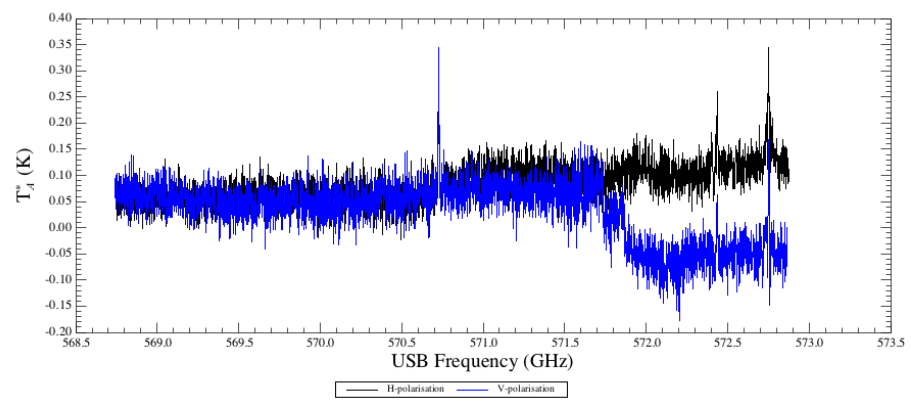

Fig. 6. The feature in the upper end of the $\mathrm{V}$ polarisation is an example of baseline distortions. Such distortions can be corrected using the task fitBaseline.

data, as well as quality, logging, and history products which can be used to identify any problems with a data-set or its processing.

This section briefly describes the additional processing that may be required for a given observation. Section 6.1 addresses corrections to specific instabilities within HIFI data, while Sects. 6.2-6.4 address more science-driven additional processing steps.

\subsection{Baseline distortions}

Common baseline distortions can take the form of platforming, residual standing waves, or other baseline instabilities: they are discussed with examples in Chap. 5.3.1 of the HB. Within the HIPE software, there were two main tools to address these instabilities: fitHifiFringe for sine-fitting of standing waves, or fitBaseline for fitting low-order polynomials to the baseline. Both of these modules are described in more detail in Chaps. 12 and 13 of the HDRG. Figures 6 and 7 show two examples of baseline instabilities that are addressed by these modules

It is best to use the Level 2 spectra since they have not been sub-band stitched. When individual spectra are to be inspected before averaging at Level 2, the non-Averaged Level 2

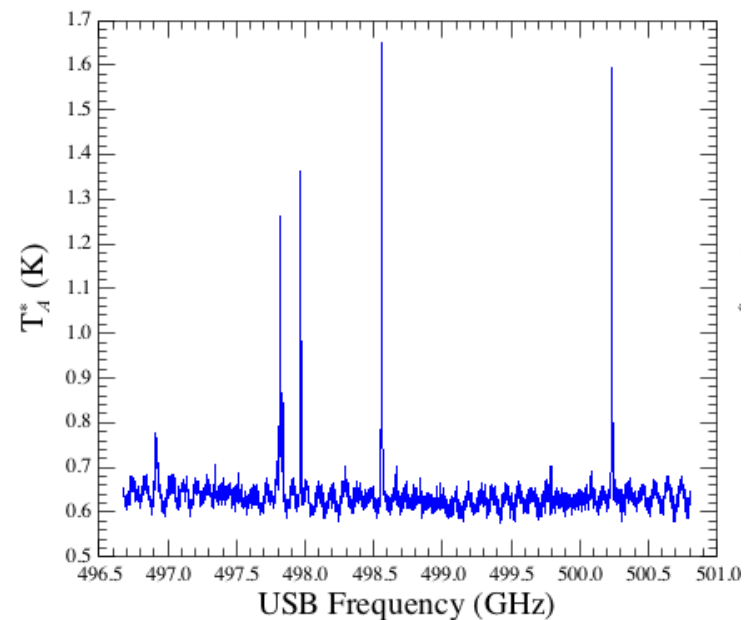

Fig. 7. Baseline ripples are an example of a standing wave in band 1a. These ripples have a period near $100 \mathrm{MHz}$ due to the internal calibration loads. The ripples can be corrected for using the task fitHifiFringe.

UPDP described in footnote 7 should be consulted. This case applies mainly to Point Mode observations since neither maps nor spectral scans often contain many repeat spectra in any given Level 2 averaging.

\subsection{Co-adding $H / V$ spectra}

Some processing steps were not taken by the standard pipeline. First, even though HIFI observed in two polarisations, the polarisations were not co-added. The pipeline did not average the polarisations for several reasons. It is possible that one polarisation was underpumped, or there were significant noise differences between the two polarisations. Another reason is that the polarisations were not perfectly spatially aligned and they could be seeing different features at slightly different positions (see Sect. 5.9 of the HB). 
However, when the data for the two polarisations are of sufficient quality and the small alignment differences are insignificant for a given object, averaging should be considered. The $\mathrm{H}$ and $\mathrm{V}$ point spectra and spectral scans can be co-added using the Level 2.5 spectra.

With maps, this is best done before the initial spectral map is created using the Level 2.5 spectra (not the spectral cubes). These spectra should first be cleaned individually in each polarisation, then sent to the spectral -cube-building software (for example the task doGridding within HIPE or map_xy within GILDAS/CLASS). The processing steps for the HIPE software are described in Chap. 15 of HDRG and in the GILDAS software package ${ }^{8}$.

The Highly-Processed Data Products available from the HSA of spectral maps in bands 6 and 7 have combined the polarisations and also inspected and removed most artefacts (see the footnote in Sect. 5.2).

\subsection{Correcting the continuum to a single-sideband scale}

Owing to the double-sideband nature of the HIFI mixers, each Level 2 spectrum is provided in both the upper- and lowersideband frequency scales. This also implies that different sideband gain ratio calibrations are applied to the respective spectra. The Level 2 spectra are therefore provided with a singlesideband intensity scale, but this is only applicable to the information held in spectral lines since they only belong to one of the two sidebands. The continuum signal, on the other hand, is present in both sidebands and is therefore not properly calibrated in Level 2 spectra. Typically, it is a factor of two higher than the real single-sideband intensity. When the continuum needs to be accurately estimated (e.g. for absorption line studies), it is possible to recover the single-sideband continuum using the following recipe (see also Sect. 5.8.4 or the $\mathrm{HB}$ ):

$T_{\mathrm{SSB}}^{\mathrm{corr}}(v)=T_{\mathrm{SSB}}(v)-\left[C_{\mathrm{DSB}}-C_{\mathrm{SSB}}\right]=T_{\mathrm{SSB}}(v)-C_{\mathrm{DSB}}\left(1-G_{\mathrm{ssb}}\right)$

where $T_{\mathrm{SSB}}(v)$ is the Level 2 or $2.5 \mathrm{USB}$ or LSB data, $C_{\mathrm{DSB}}$ is the estimated continuum from the Level 2 or 2.5 product, and $G_{\text {ssb }}$ is the sideband gain applied to that sideband which can be found in the product header under the keyword usbGain or lsbGain depending on which sideband was used. We note that this correction assumes that the continuum emission does not differ between the two sidebands. We also note that this is not a deconvolution since spectral features in both sidebands may be present in the resulting spectrum.

\subsection{Converting the data into $T_{M B}$ or Jy}

The antenna temperature scale can also be easily changed into main-beam temperature $T_{\mathrm{MB}}$ or into Jansky. For the former, assuming a source filling the telescope main beam, the $T_{\mathrm{MB}}$ is simply given by:

$T_{\mathrm{MB}}=\frac{\eta_{\mathbf{l}}}{\eta_{\mathrm{MB}}} T_{\mathrm{A}}^{*}$

where $\eta_{l}$ is the forward efficiency (taken to be 0.96 for all HIFI bands), and $\eta_{\mathrm{MB}}$ is the main-beam efficiency for a source that fills the beam. This gain is found in the product header under beamEff.

On the other hand, the conversion from $T_{\mathrm{A}}^{*}$ into Janskys requires making an assumption of the source size, as beam filling factors enter the equation (Mueller et al. 2014). Analytical

\footnotetext{
8 http://www.iram.fr/IRAMFR/GILDAS/
}

formulae exist for perfectly Gaussian beams and simple source morphologies (e.g. Gaussian, top hat). A dedicated HIPE task, convertK $2 \mathrm{Jy}$, exists that allows performing this conversion with one of the simple source morphologies mentioned earlier, or even provide an ad hoc source brightness distribution. The task uses the full 2D model of the HIFI beams. The conversion factor for a perfectly pointed point-source is given in Table 4. Further details on scale conversion can be found in Sect. 5.8.5 of the HB.

\section{Discussion}

\subsection{Project lifespan}

Parts of the HIFI pipeline were developed at a time well in advance of actual use. The Levels 0 and 0.5 pipelines were in heavy use during instrument level testing before launch. The Level 2 pipeline, on the other hand, was initially a placeholder that averaged all Level 1 spectra. Keeping the steps well modularised helped the development, since any additional new step could be introduced into an existing pipeline script. In the end, this approach allowed the pipeline to mature as the understanding of the HIFI instrument and its data matured.

Early development had the risk of introducing tacit and hard-to-correct assumptions about the instrument, satellite, calibrations, and the data products themselves. Perhaps, the most important early development was the definition of the data products. Data products reflect the instrument that produced them and the way in which the instrument was used. For HIFI, the main data features were instrument counts and frequency scales as functions of time. For HIFI, both the counts and the frequency scales could change with time. The implementation of the initial HIFI products was two-dimensional spectra. Data products also need to reflect the science uses, however. The final products must be usable by the community at large. Usability means either that the format of the product is standard enough to work with standard analysis software, or that there is available speciality software that understands the format of the new products.

At the beginning of the project, it was tacitly assumed that the HCSS/HIPE user interface would continue well beyond post-operations. Since there was no plan for continued maintenance, the assumption went further to hope that eventually the HCSS/HIPE software would become standard analysis software. Going into post-operations, it looked doubtful that HIPE would continue to be supported beyond the end of the HSC. This meant that a better support of other data formats was required. For heterodyne spectroscopy, an exporter to the GILDAS/CLASS data format was developed mainly to support the HIFI consortium. During post-operations, Level 2.5 HIFI data products were introduced which were a simple reformatting of the PointSpectrum away from the HifiSpectrumDataset (2D spectra) to a more standard flux-vs.-frequency format (SimpleSpectrum). However, giving the restrictions of the HCSS/HIPE software, even this final format involved added complexity to a conceptually simple data product.

Projects such as Herschel must address not just the final archiving of the data products, but must also retain their utility. Usability can be achieved by defining products that conform to standard formats (if they exist) or by maintaining specialist software over the long term and by providing complete documentation of the data formats used in the project. This point is further discussed below. 


\subsection{Herschel context}

The Herschel ground segment made the decision to use common open source software for all instruments. Since no common software package existed that supported all three instruments, a new environment had to be created. This environment had to support the instrument engineers, bulk data processing, and the Herschel user community, but also data products from the different instruments. The resulting software was the so-called Herschel Common Software System (HCSS) with HIPE providing the user interface.

The HCSS was a novel concept with far-reaching implications. The requirement was for open and common software for all Herschel instruments. This further implied that the HCSS should operate on machines running under any "common" operating system and not use any proprietary software. Finally, the FITS table format was chosen to save and restore products.

The requirement that all three Herschel instruments use HCSS meant that all instruments were using software different than what each science community used and indeed, since data product formats reflected both the instrument and the software, the HCSS products were often not directly usable in standard analysis software. To remedy this situation, the ground segment also took on the task of providing analysis software within the HCSS and a user environment. This extra effort required additional support from ESA in the form of software-development contracts.

The HCSS/HIPE provided the required environment for all the instrument teams to calibrate and pipeline process the data of their instrument. It also provided the necessary data management (tasks reading from the HSA) or inspecting contents of an observation, and many novel innovations required to support the diverse data products that Herschel produces. In the end, HCSS/HIPE was able to process, display and manipulate images, spectra, and spectral cubes. Unfortunately, the user software (HIPE) was initially released before it had reached sufficient maturity and user-friendliness as a scientific data analysis package, and, as a consequence, never gained wide user acceptance by the science community.

The HIPE software will not be maintained beyond the end of Herschel post-operations (although the final legacy version will be provided via a virtual machine). One of the consequences of this is that many HIFI products that are easily accessible from within HIPE needed to be recast into stand-alone formats that can be accessed independent of HIPE from the HSA and/or the HSC user pages. The stand-alone products are noted in earlier sections as well as in the HB. Currently, there are software packages other than HIPE to deal with HIFI products. Furthermore, all Herschel data products are in the FITS binary table format, which describes the data accurately and sufficiently ensuring that the HIFI products will remain accessible and interpretable for years to come.

\subsection{How well did the pipeline perform}

With the goal of removing instrument artefacts and preparing scientifically useful data, the HIFI pipeline systematically processed all HIFI observations to the highest processing level, and by processing in modular steps, facilitated data analysis. Guaranteeing completely artefact-free final products, however, remained a challenge. Ground-based observatories rely heavily on the observers making the final call about their data. To achieve the science goals, the observer decides which data to clean and which data are filtered out. The final steps of ground-based processing are interactive.

There are two fundamental problems with the final steps of pipeline processing. The first is that the pipeline can only make a reasonable estimate as to the overall science goals that resulted in the given observation. Thus maps, for example, are created with standard parameters that may not be the optimum for a given science question. Secondly, despite the stable Herschel environment, the HIFI data still suffered from residual baseline artefacts that were due to imperfect bandpass calibration. These problems finally had to be addressed (interactively or otherwise) outside of the pipeline through the intervention of instrument experts, and were made available to the community via the HSA as highly processed data products.

\section{Summary}

This article presented the concepts of the HIFI pipeline. The main concepts of the pipeline are listed below.

- One pipeline for all HIFI observations that populates the Herschel Science Archive.

- Process HIFI data in levels of increasing uncertainty retaining results from all processing levels.

- Provide a pipeline that produces the same HIFI products either autonomously within the Herschel Standard Product Generation environment or interactively in the Herschel Interactive Processing Environment.

- Assess processing quality for each processing step.

The HIFI pipeline has supported analysis of HIFI data since before the launch of Herschel. The modular design was able to cope with the many changes that faced the 15-yr lifetime and provided a smooth transition of the data processing from early instrument models through post-mission operations. Along the way, calibrations were updated and new calibrations were introduced. Entirely new processing ideas were developed and employed. New processing levels were introduced allowing for significantly different product types to be supported.

The HIFI pipeline processed all HIFI observing requests and calibrations taken during the Herschel mission and performed initial quality assessments. The Herschel Legacy Science Archive is populated with the pipelined data and quality results. We described here are the processing steps that the products in the HSA underwent and the important quality measures taken.

This article provides a context on how to understand the various data products and quality assessments provided with HIFI data as well as suggestions on how to inspect the HIFI data products themselves. Along with presenting details on the pipeline levels, we also briefly described some of the limitations to automatic data-processing and additional processing steps beyond the pipeline.

Acknowledgements. HIFI has been designed and built by a consortium of institutes and university departments from across Europe, Canada and the United States under the leadership of SRON Netherlands Institute for Space Research, Groningen, The Netherlands, and with major contributions from Germany, France and the US Consortium members are: Canada: CSA, University of Waterloo; France: CESR, LAB, LERMA, IRAM; Germany: KOSMA, MPIfR, MPS Ireland, NUI Maynooth; Italy: ALI, IFSI-INAF, Osservatorio Astrofisico di Arcetri-INAF; The Netherlands: SRON, TUD; Poland: CAMK, CBK; Spain: Observatorio Astron Nacional (IGN), Centro de Astrobiologia (CSIC-INTA). Sweden: Chalmers University of Technology - MC2, RSS \& GARD; Onsala Space Observatory; Swedish National Space Board, Stockholm University Stockholm Observatory; Switzerland: ETH Zurich, FHNW; USA: Caltech, JPL, NHSC. 
R. F. Shipman et al.: Data processing pipeline for Herschel HIFI

\section{References}

Bardeau, S., \& Pety, J. 2015, The new CLASS Associated Arrays, Memo 20154, IRAM

Bardeau, S., Teyssier, D., Rabois, D., \& Pety, J. 2015, Importing Herschel-FITS into CLASS, Memo 2015-3, IRAM

Beaulieu, S., \& Teyssier, D. 2017, HIFI Products Explained, HERSCHEL-HSCDOC-2174 (ESA)

Belgacem, M., Ravera, L., Caux, E., Caïs, P., \& Cros, A. 2004, New Astron., 9 43

Calabretta, M. R., \& Greisen, E. W. 2002, A\&A, 395, 1077

Comito, C., \& Schilke, P. 2002, A\&A, 395, 357

de Graauw, T., Helmich, F. P., Phillips, T. G., et al. 2010, A\&A, 518, L6

Herpin, F., \& Teyssier, D. 2003, Frequency calibration framework document, Tech. Rep. L3AB/HIFI/CAL/2003-02

Kester, D., Avruch, I., \& Teyssier, D. 2014, in Bayesian Inference and Maximum Entropy Methods in Science and Engineering, AIP Conf. Proc., 1636, 62

Kester, D., Higgins, R., \& Teyssier, D. 2017, A\&A, 599, A115

Kidger, M. R. 2013, Herschel-Spot (HSpot) Users' Guide: Herschel Observation Planning Tool, v5.2 for HSpot 7.0
Kutner, M. L., \& Ulich, B. L. 1981, ApJ, 250, 341

Morris, P. 2011, HIFI AOT Observing Mode Release and Performance Notes, Tech. Rep., SRON

Mueller, M., Jellema, W., Olberg, M., Moreno, R., \& Teyssier, D. 2014, The HIFI Beam: Release 1 - Release Note for Astronomers, Tech. Rep. HIFIICC-RP-2014-001, SRON Groningen

Ossenkopf, V. 2003, The HIFI intensity calibration framework, Tech. Rep., 442, ALMA Memo Series

Ossenkopf, V. 2008, A\&A, 479, 915

Ossenkopf, V. 2015, The propagation of systematic uncertainties in the intensity calibration for HIFI, Tech. Rep., University of Cologne

Ott, S. 2010, in Astronomical Data Analysis Software and Systems XIX, eds. Y. Mizumoto, K.-I. Morita, \& M. Ohishi, ASP Conf. Ser., 434, 139

Pilbratt, G. L., Riedinger, J. R., Passvogel, T., et al. 2010, A\&A, 518, L1

Roelfsema, P. R., Helmich, F. P., Teyssier, D., et al. 2012, A\&A, 537, A17

Schieder, R. T., Siebertz, O., Gal, C., et al. 2003, in Millimeter and Submillimeter Detectors for Astronomy, eds. T. G. Phillips, \& J. Zmuidzinas, SPIE Conf. Ser., 4855, 290

Teyssier, D., Avruch, I., Beaulieu, S., et al. 2017, HIFI Handbook, HERSCHELHSC-DOC-2097 


\section{Appendix A: Level 0 to 2.0 pipeline diagrams}

From Level 0 to Level 2.0, various steps were taken in the pipeline. The following flow diagrams, Figs. A.1 to A.5, give a global view of the steps specific to each level. See Sect. 4 for a brief description of each level.
The pipeline tasks are indicated in the light blue rectangles. Calibration products are shown in dark green. Task optional inputs are indicted with a red rhombus pointing to that task. A red rhombus pointing to the pipeline flow indicates a quality-check step.

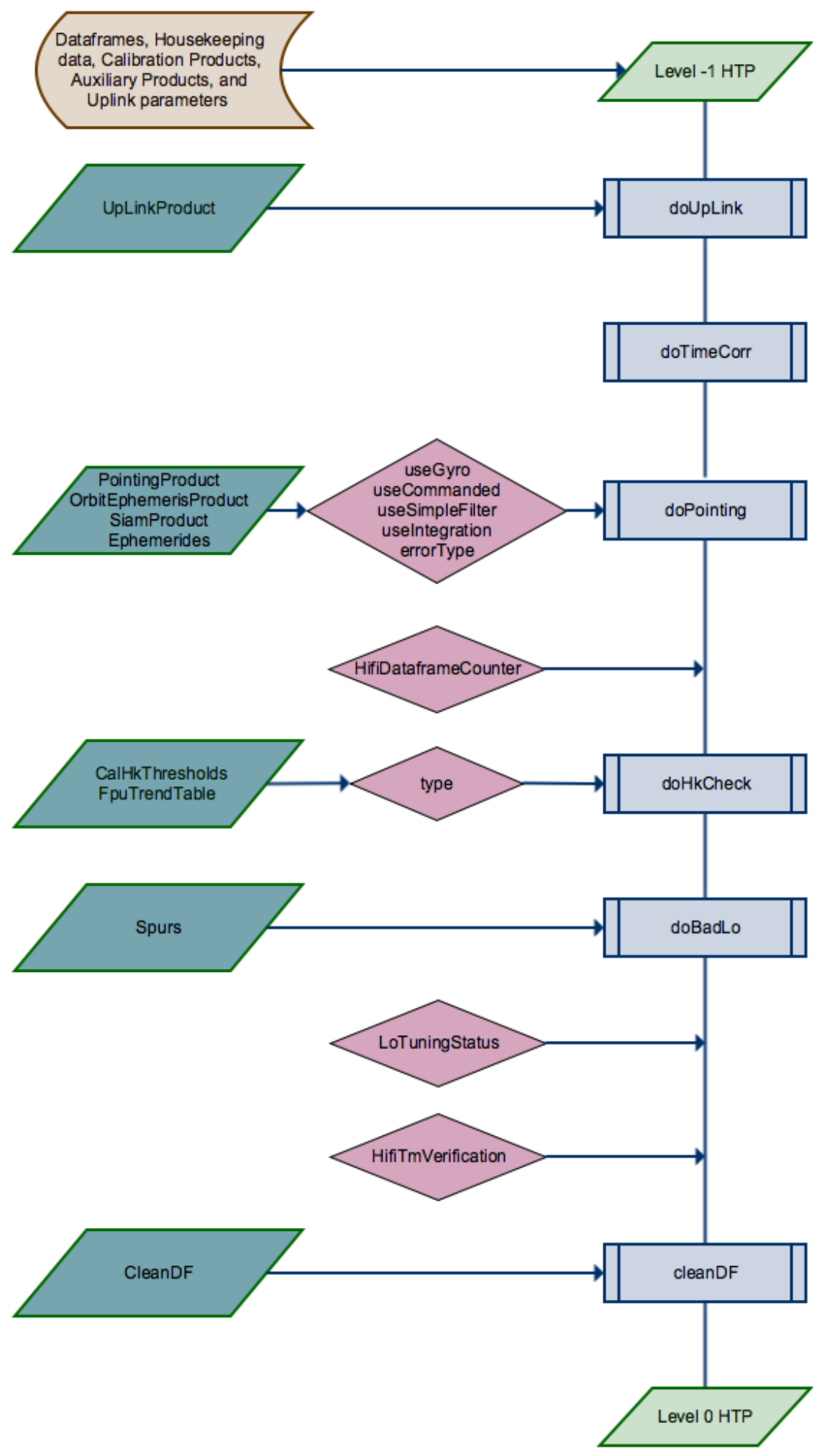

Fig. A.1. Level 0 pipeline diagram. The different shapes and colours have specific meanings. Pipeline tasks are identified in light blue rectangles. The beginning and ending products are shown as light green parallelograms. Calibration items are the dark green parallelograms. Calibration can be either obtained from the calibration tree or created by a pipeline step. Task options are identified within red rhombuses connected to a task. A red rhombus connected directly to the pipeline indicates a quality-check step. 


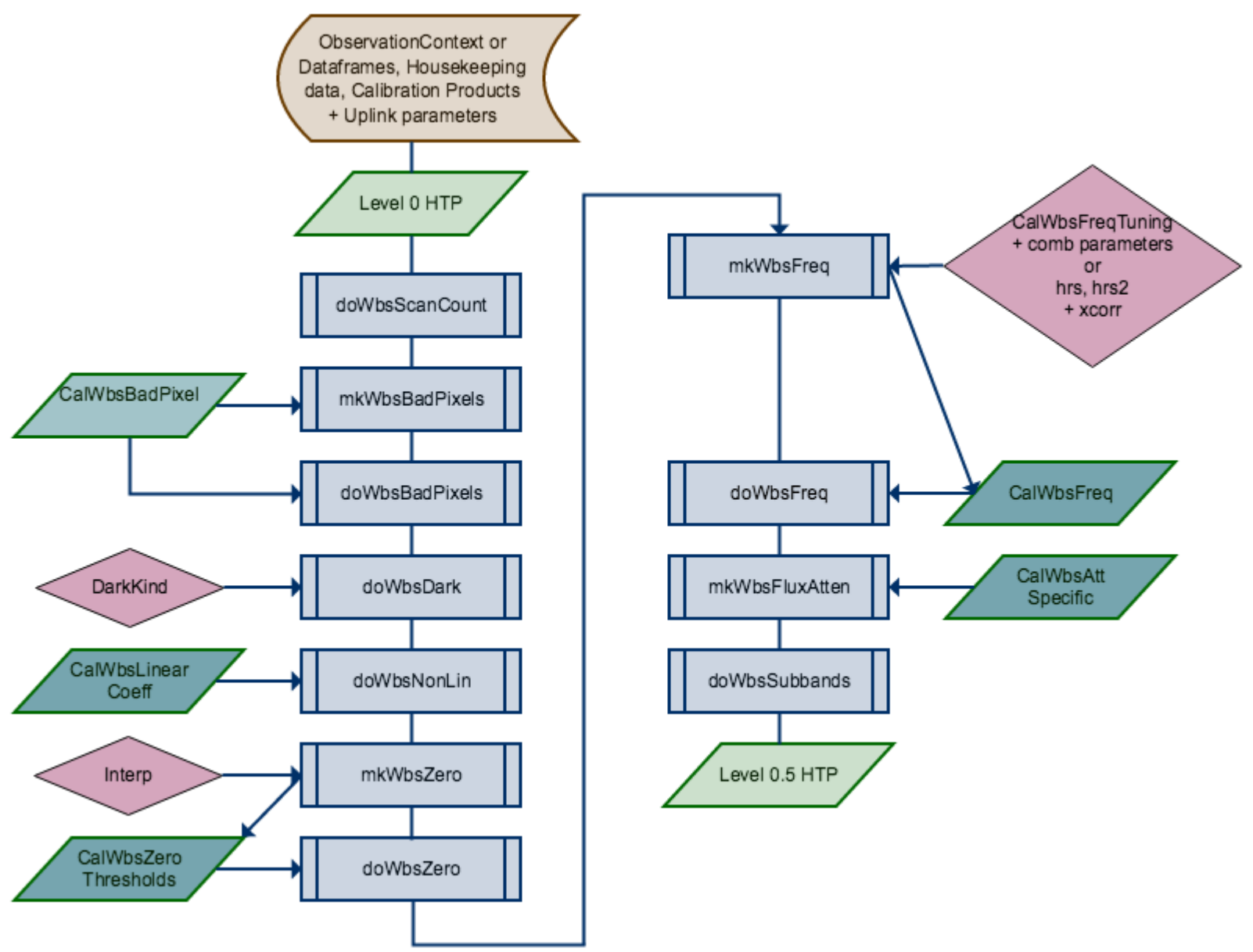

Fig. A.2. Level 0.5 pipeline diagram for the WBS. The input to this part of the pipeline is Level 0 WBS data and calibration data in the ObservationContext. The symbols have the same meaning as for the Level 0 pipeline in Fig. A.1. 


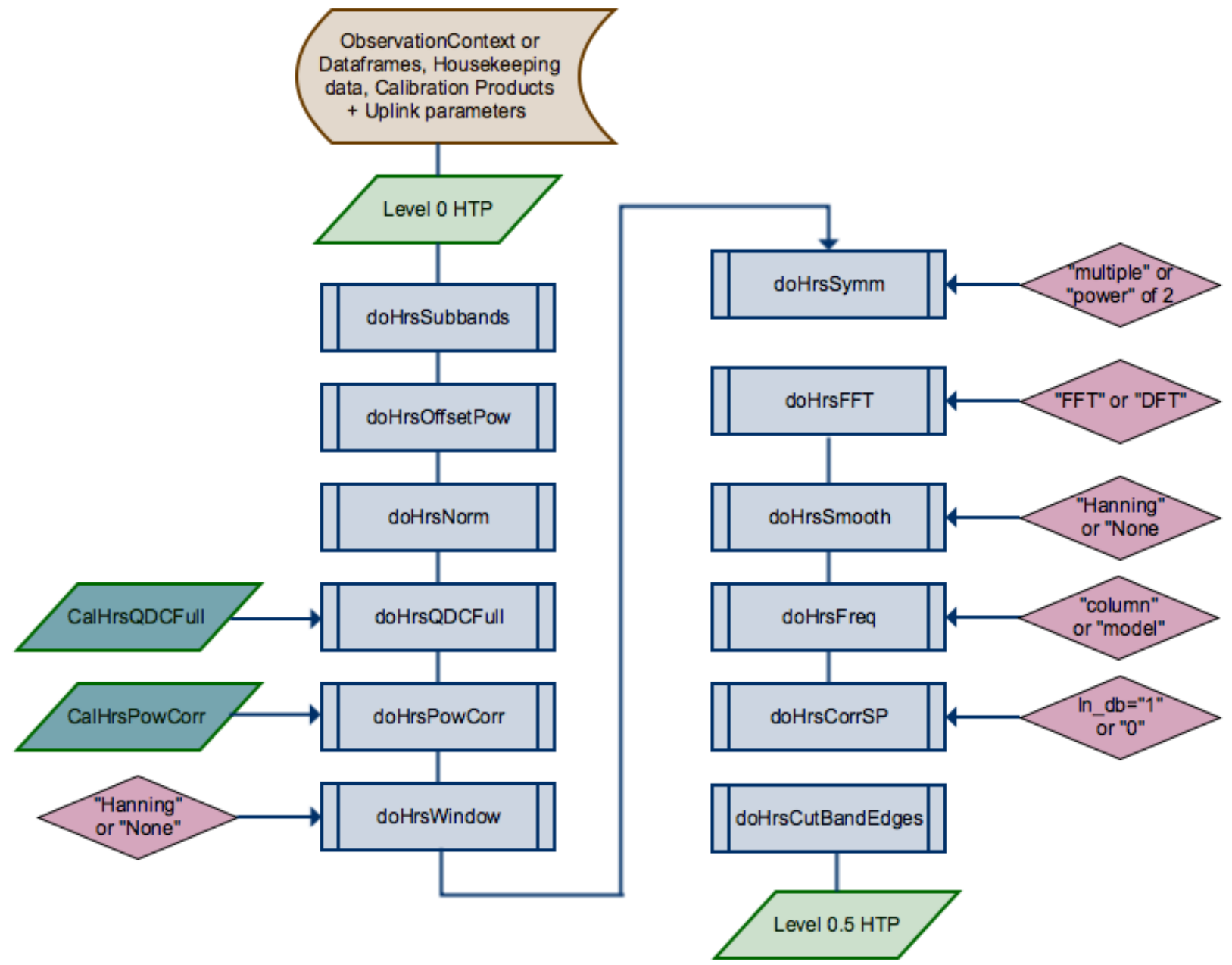

Fig. A.3. Level 0.5 pipeline diagram for the HRS. The input to this part of the pipeline is Level 0 HRS data and calibration data in the ObservationContext. The symbols have the same meaning as for the Level 0 pipeline in Fig. A.1. 


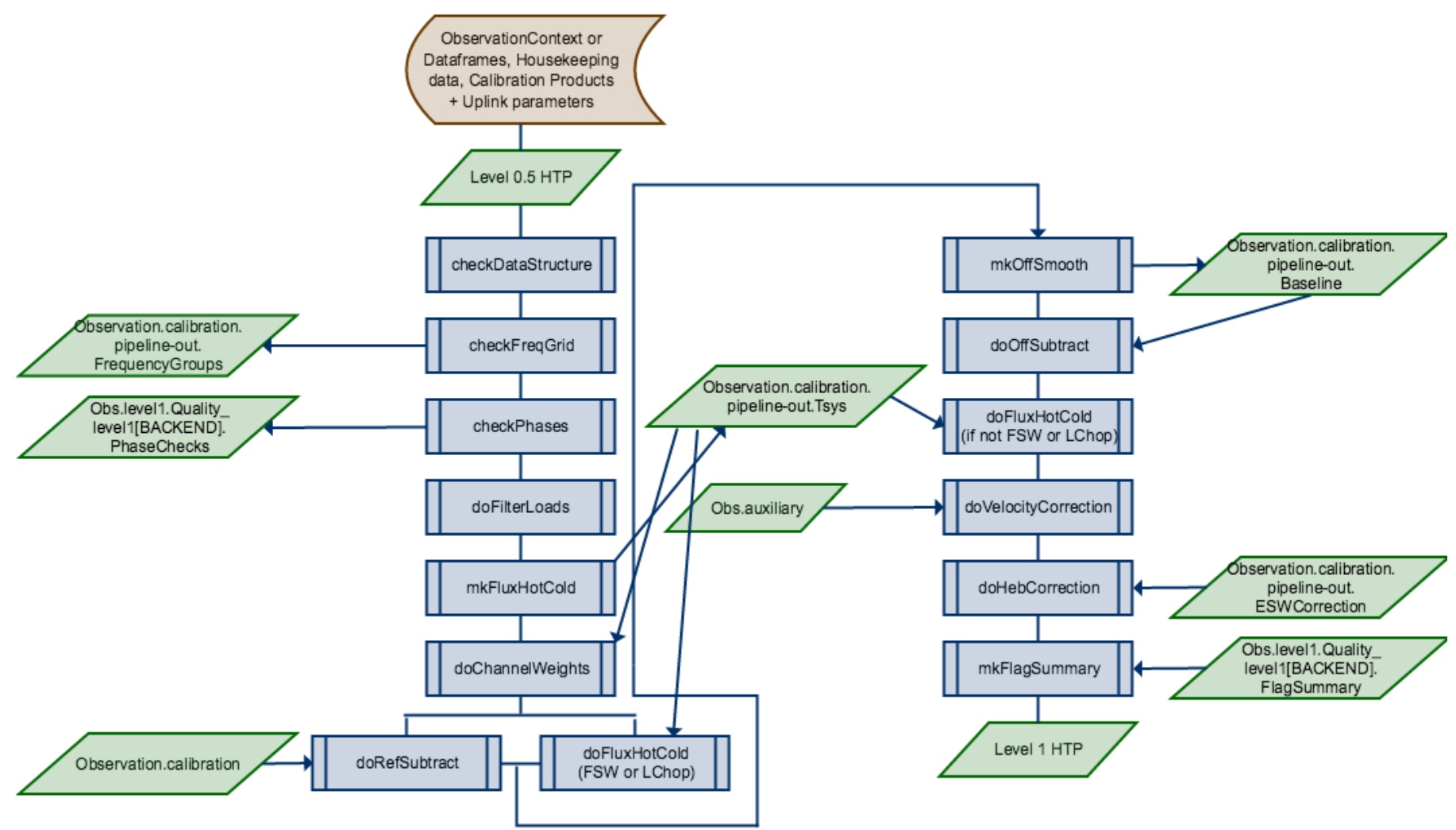

Fig. A.4. Level 1 pipeline flow diagram. The Level 1 pipeline uses the results of the Level 0.5 pipelines and processes the two backend in the same fashion. This and the Level 2 pipelines are also often called the GenericPipeline. The Level 0.5 results were removed from the ObservationContext to save disk space. The symbols and colours have the same meaning as for the Level 0 pipeline in Fig. A.1. 


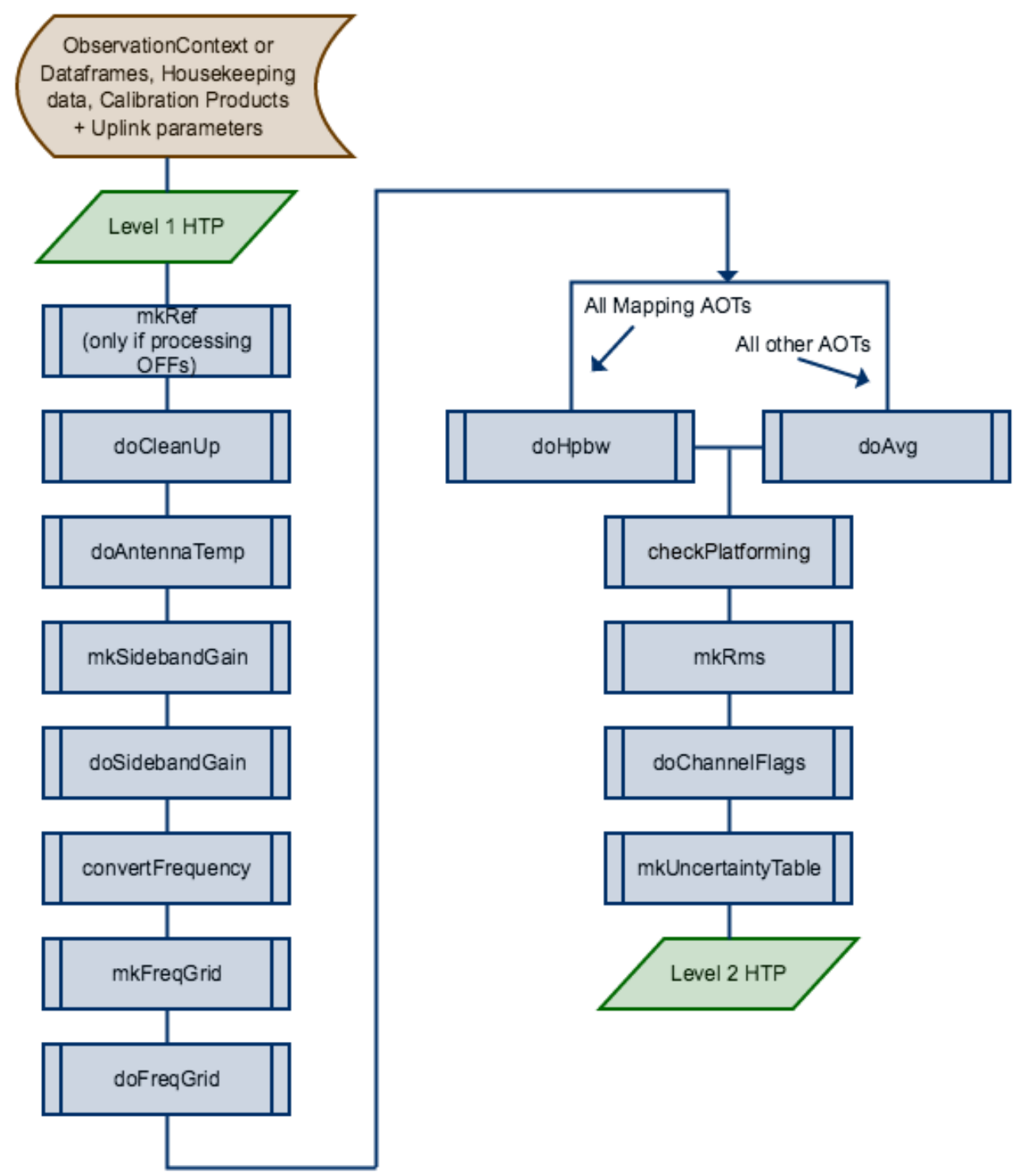

Fig. A.5. Level 2 pipeline diagram. The Level 2 pipeline uses the results of the Level 1 pipeline. The symbols and colours in the figure have the same meaning as for the Level 0 pipeline in Fig. A.1. 